\title{
苯基吡唑氧基丙酸衍生物的设计、合成及其对水稻纹枯病的杀菌活性
}

\author{
于福强 $a, b$ 关爱莹 ${ }^{*}, b$ 孙旭峰 ${ }^{b}$ 李慧超 ${ }^{b}$ 李小武 $*, a$ \\ ( ${ }^{a}$ 东北大学材料科学与工程学院 材料物理与化学系 材料各向异性与织构教育部重点实验室 沈阳 110819) \\ ( ${ }^{b}$ 沈阳中化农药化工研发有限公司 新农药创制与开发国家重点实验室 沈阳 110021)
}

\begin{abstract}
摘要 水稻纹枯病是水稻的三大病害之一, 发病后可造成水稻严重减产, 使用杀菌剂是使水稻免遭病害侵扰的有效途 径之一. 由于杀菌剂使用一段时间后病菌不可避免地会对其产生抗药性，因此需要不断开发结构新颖的化合物. 采用 中间体衍生化方法, 设计并合成了 25 个结构新颖的苯基吡唑氧基丙酸衍生物. 所有化合物都进行了离体生物活性测 试, 并讨论了构效关系. 生物活性测试数据表明, 结构新颖的苯基吡唑氧基丙酸衍生物对水稻纹枯病菌均具有良好的 抑制作用, 其中 2-((1-(4-氯苯基)- $1 H$-吡唑-3-基)氧基)- $N$-(4-硝基苯基)丙酰胺(17) $\left(\mathrm{EC}_{50}=1.05 \mathrm{mg} / \mathrm{L}\right.$ )和 2-((1-(4-氯苯 基)- $1 H$-吡唑-3-基)氧基)- $N$-(吡啶-2-基)丙酰胺(22) ( $\mathrm{EC}_{50}=1.02 \mathrm{mg} / \mathrm{L}$ )对水稻纹枯病菌的杀菌活性与对照药物戊唑醇 $\left(\mathrm{EC}_{50}=1.02 \mathrm{mg} / \mathrm{L}\right)$ 相当; 2-((1-(4-氯苯基)-1 $1 H$-吡唑-3-基)氧基)- $N$-(3,4-二氯苯基)丙酰胺(20) $\left(\mathrm{EC}_{50}=0.95 \mathrm{mg} / \mathrm{L}\right.$ )活性略优 于商品化对照药剂龙唑醇 $\left(\mathrm{EC}_{50}=1.02 \mathrm{mg} / \mathrm{L}\right)$.
\end{abstract}

关键词＼cjkstart苯基吡唑氧基丙酸衍生物; 中间体衍生化; 杀菌活性; 水稻纹枯病; 构效关系

\section{Design, Synthesis and Fungicidal Activity against Rhizoctonia solani of New Phenylpyrazoloxyl Propionic Acid Derivatives}

\author{
Yu, Fuqiang ${ }^{a, b} \quad$ Guan, Aiying $^{*, b} \quad$ Sun, Xufeng ${ }^{b} \quad$ Li, Huichao $^{b} \quad$ Li, Xiaowu ${ }^{*, a}$ \\ $\left({ }^{a}\right.$ Key Laboratory for Anisotropy and Texture of Materials, Ministry of Education, Department of Materials Physics and \\ Chemistry, School of Material Science and Engineering, Northeastern University, Shenyang 110819) \\ $\left({ }^{b}\right.$ State Key Laboratory of the Discovery and Development of Novel Pesticide, Shenyang Sinochem Agrochemicals \\ R\&D. Co. Ltd., Tiexi District, Shenyang 110021)
}

\begin{abstract}
Rice sheath blight disease, one of three most important diseases in rice, can lead to crop production loss seriously. However, the application of fungicides is an effective way to prevent rice from diseases. After being used for a period of time, the plant pathogenic fungi may develop resistance to chemical fungicide, so it is necessary to discover compounds with novel structures and further to address the problem of fungicide resistance. By employing the intermediate derivatisation method (IDM), twenty-five new phenylpyrazoloxyl propionic acid derivatives were designed and synthesized. The biological activities of title compounds were tested against Rhizoctonia solani and their structure-activity relationships (SAR) were discussed as well. The preliminary bioassay results displayed that some compounds exhibited an excellent fungicidal activity against $R h i-$ zoctonia solani, especially 2-((1-(4-chlorophenyl)-1H-pyrazol-3-yl)oxy)- $N$-(4-nitrophenyl)propanamide (17) $\left(\mathrm{EC}_{50}=1.05 \mathrm{mg} /\right.$ $\mathrm{L})$ and 2-((1-(4-chlorophenyl)-1H-pyrazol-3-yl)oxy)- $N$-(pyridin-2-yl)propanamide (22) $\left(\mathrm{EC}_{50}=1.02 \mathrm{mg} / \mathrm{L}\right)$ showing a comparable activity to the commercial contrast tebuconazole $\left(\mathrm{EC}_{50}=1.02 \mathrm{mg} / \mathrm{L}\right)$, and 2-((1-(4-chlorophenyl)-1H-pyrazol-3-yl)oxy)- $N$-(3,4-dichlorophenyl)propanamide $(\mathbf{2 0})\left(\mathrm{EC}_{50}=0.95 \mathrm{mg} / \mathrm{L}\right)$ is a little more active than tebuconazole $\left(\mathrm{EC}_{50}=1.02\right.$ $\mathrm{mg} / \mathrm{L})$.
\end{abstract}

Keywords phenylpyrazoloxyl propionic acid derivatives; intermediate derivatization method; fungicidal activity; Rhizoctonia solani; structure-activity relationship

\section{Introduction}

The rice is one of important major food crops, which is planted widely around the world with harvested area of 160.1 million ha in $2016,{ }^{[1]}$ to guarantee the food provision for over half the global population. ${ }^{[2,3]}$ Rice sheath blight

* Corresponding authors. E-mail: guanaiying@sinochem.com; xwli@mail.neu.edu.cn

Received July 4, 2018; revised September 5, 2018; published online October 14, 2018.

Project supported by the Liaoning Provincal Science and Technology Plan Projects (No. 2015306001).

辽宁省科技计划基金(No. 2015306001)资助项目. 
(Rhizoctonia solani Kühn) is one of three most important diseases in rice, which can cause even $40 \% \sim 50 \%$ production loss in crops per year worldwide. ${ }^{[4,5]}$ There are many ways to prevent and control this destructive disease; however, the application of chemical pesticides is one of the most effective ways. At present, the existing chemical pesticides for control of rice sheath blight are mainly triazoles and strobilurins such as tebuconazole, ${ }^{[6,7]}$ pyraclostrobin ${ }^{[8,9]}$ and azoxystrobin. ${ }^{[10,11]}$ However, after fungicide is used for a period of time, the fungi will develop resistance to it. As been reported by the Fungicide Resistance Action Committee (FRAC), the known target sites had already developed medium to high resistance risk to most triazole and strobilurin fungicides. ${ }^{[12]}$ Furthermore, along with the increase of people's living standard, the demand for quality, quantity and safety of grain increases daily. So it urgently needed to discover and develop new fungicides with an innovated structure to protect rice from rice sheath blight and further to facilitate resistance management.

To the best of our knowledge, aryloxy carboxylic acid compounds are mostly known as herbicides in agricultural field, such as aryloxyphenoxypropionate (APP) like metamifop ${ }^{[13,14]}$ and cyhalofop butyl. ${ }^{[15,16]}$ In recent years, this kind of compounds is being paid more and more attention $^{[17]}$, and it is also found to have good inhibitory effects on fungus and insects. ${ }^{[18 \sim 20]}$ Nevertheless, their application as fungicides for control of rice sheath blight has not been reported. On the other hand, pyraclostrobin is a broad spectrum fungicide, and it has a good control effect on cucumber powdery mildew, downy mildew, banana black star, leaf spot, sclerotia and sheath blight. ${ }^{[21]}$ In order to develop new compounds for preventing and controlling rice sheath blight, with the guidance of intermediate derivatisation method (IDM), ${ }^{[22 \sim 24]}$ mimicking the skeleton of metamifop, we designed and synthesized a series of new phenylpyrazoloxyl derivatives by retaining the fragment of propanamide and replacing (benzo $[d]$ oxazol-2-yloxy)phenyl with phenylpyrazolyl of pyraclostrobin (Scheme 1). The detailed syntheses, bioassay results and structure- activity relationships of these derivatives were discussed below.

\section{Results and discussion}

\subsection{Synthesis}

As shown in Scheme 2, twenty-five compounds were synthesized. The synthesized compounds were characterized by ${ }^{1} \mathrm{H}$ NMR, ${ }^{13} \mathrm{C}$ NMR, IR, MS and elemental analyses. All spectral and analytical data were consistent with the assigned structures. The chemical structure of compound $\mathbf{8}$ was unequivocally determined by single-crystal X-ray crystallography (Figure 1). ${ }^{[25]}$

\subsection{Structure-activity relationship}

\subsubsection{Discovery of lead compound}

Originally, to verify whether phenylpyrazoloxyl propionic acid derivatives designed have fungicidal activity against Rhizoctonia solani or not, some representative alkyl and cycloalkyl amines were employed to synthesize compounds $\mathbf{1} \sim \mathbf{4}$ (Table 1). Fortunately, all of synthesized compounds showed certain fungicidal activity. These results suggested that the groups linked to nitrogen atom

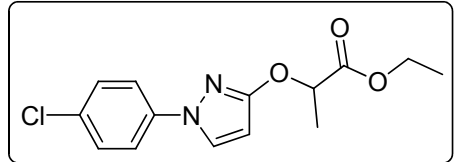

A

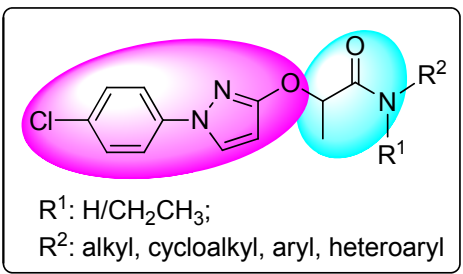

Compounds $1 \sim 25$

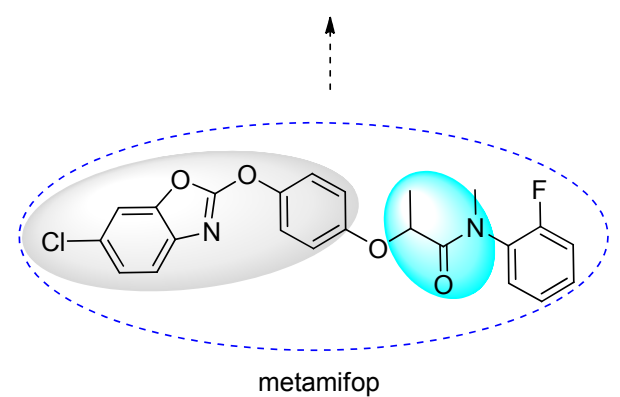

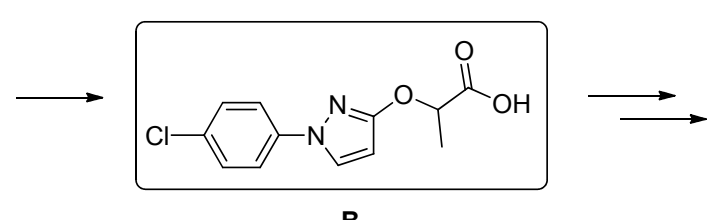

B
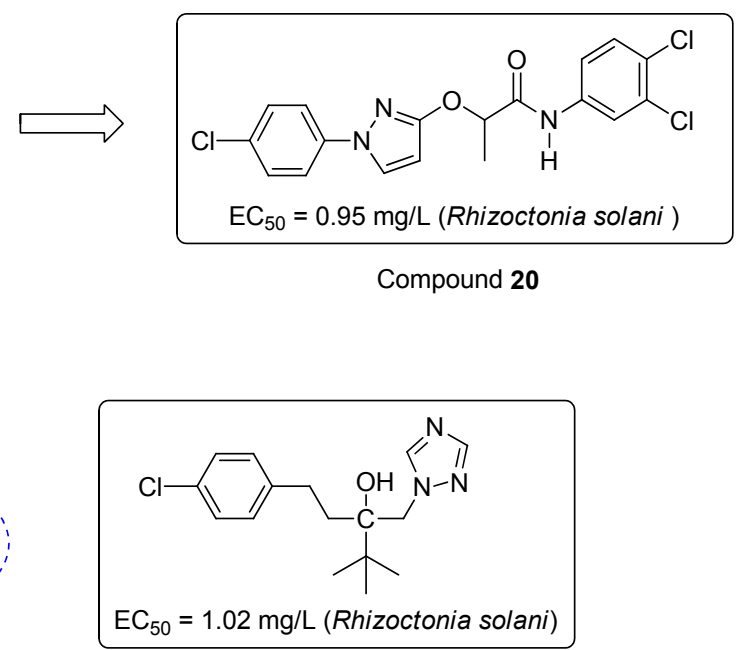

tebuconazole

Scheme 1 Design strategy of new phenylpyrazoloxyl propionic acid derivatives 


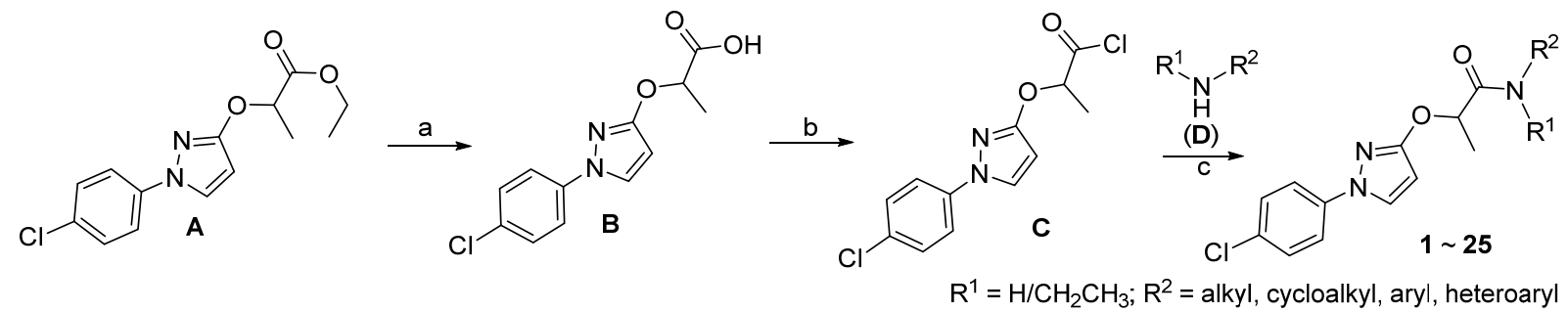

Reaction conditions: (a) $\mathrm{NaOH}, \mathrm{CH}_{3} \mathrm{CH}_{2} \mathrm{OH}, 60{ }^{\circ} \mathrm{C}, 2 \mathrm{~h}$; (b) $\mathrm{SOCl}_{2}$, reflux, 3 h; (c) $\mathrm{Et}_{3} \mathrm{~N} / \mathrm{CH}_{2} \mathrm{Cl}_{2}$, r.t., 2 h

Scheme 2 Synthetic route of compounds $1 \sim 25$

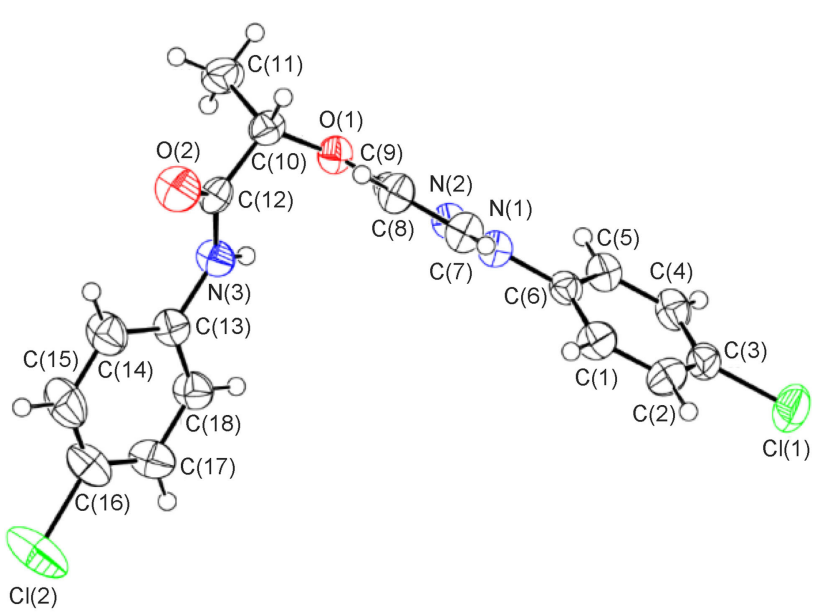

Figure 1 X-ray single-crystal diffraction of compound $\mathbf{8}$

maybe play an important role in the interaction of active molecules with targets. Furthermore, it seemed that a larger cyclic moiety and mono-substituted nitrogen atom will benefit to enhance fungicidal activity.

Table 1 Fungicidal activity against Rhizoctonia solani of phenylpyrazoloxyl propionic acid derivatives $\mathbf{1} \sim \mathbf{8}$

\begin{tabular}{cclcc}
\hline Compd. & \multicolumn{2}{c}{$\mathrm{R}^{1}$} & \multicolumn{1}{c}{$\mathrm{R}^{2}$} & $\begin{array}{c}\mathrm{EC}_{50} / \\
\left(\mathrm{mg} \bullet \mathrm{L}^{-1}\right)\end{array}$ \\
\hline $\mathbf{1}$ & $\mathrm{CH}_{2} \mathrm{CH}_{3}$ & $\mathrm{CH}_{2} \mathrm{CH}_{3}$ & 21.74 & $17.79 \sim 26.56$ \\
$\mathbf{2}$ & $\mathrm{H}$ & $\left(\mathrm{CH}_{2}\right)_{3} \mathrm{CH}_{3}$ & 19.44 & $16.15 \sim 23.39$ \\
$\mathbf{3}$ & $\mathrm{H}$ & Cyclopropyl & 13.09 & $10.14 \sim 16.90$ \\
$\mathbf{4}$ & $\mathrm{H}$ & Cyclopentyl & 12.90 & $9.87 \sim 16.85$ \\
$\mathbf{5}$ & $\mathrm{H}$ & Phenyl & 12.97 & $9.96 \sim 16.87$ \\
$\mathbf{6}$ & $\mathrm{H}$ & ortho-Chlorophenyl & 17.43 & $13.66 \sim 22.23$ \\
$\mathbf{7}$ & $\mathrm{H}$ & meta-Chlorophenyl & 47.22 & $24.25 \sim 91.97$ \\
$\mathbf{8}$ & $\mathrm{H}$ & para-Chlorophenyl & 8.58 & $6.51 \sim 11.30$ \\
\hline${ }^{a}$ Confidence limit. & & &
\end{tabular}

Then we turned our attention to change $\mathrm{R}^{2}$ when $\mathrm{R}^{1}$ was fixed as $\mathrm{H}$ by introducing larger phenyl (Table 1). Compound $\mathbf{5}$ was synthesized and displayed equal activity to compound $4\left(\mathrm{EC}_{50}=12.97 \mathrm{mg} / \mathrm{L}\right.$ versus $\left.12.90 \mathrm{mg} / \mathrm{L}\right)$, which indicated that phenyl $\left(\mathrm{R}^{2}\right)$ seems to be helpful for the intact molecule to elaborate its fungicidal activity against Rhizoctonia solani. At the same time, considering a wider modifiable space based on benzene ring than cycloalkyl, using compound $\mathbf{5}$ as the starting point, our attention was paid to the preparation and evaluation of other analogous around compound $\mathbf{5}$ by changing substituents on benzene ring.

Firstly, to evaluate the position effect of the substituent on fungicidal activity, compounds $\mathbf{6} \sim \mathbf{8}$ were synthesized using chlorine atoms as probes. The results indicated that when the single chlorine was attached to ortho-position (compound 6) or meta-position (compound 7), there was no any contribution to the improvement of activity $\left(\mathrm{EC}_{50}=\right.$ 17.43 and $47.22 \mathrm{mg} / \mathrm{L}$, respectively). On the contrary, when the single chlorine was located at para-position of phenyl ring (compound 8), we found surprisingly a significant improvement in fungicidal activity $\left(\mathrm{EC}_{50}=8.58\right.$ $\mathrm{mg} / \mathrm{L})$. The result suggested that single substitution at $p a-$ ra-position probably has a positive effect on bioactivity. Encouraged by this satisfying finding, we took compound 8 as the lead compound and continued to modify the substituents.

\subsubsection{Structural modifications around lead compound}

Subsequently, the electronic properties, spatial characteristics, and numbers of substituents were discussed.

Firstly, the single chlorine on para-position was substituted with other halogen atoms (Table 2). Compounds 9 , $\mathbf{1 0}$ and 11 were synthesized, and they were all much less efficacious with $\mathrm{EC}_{50}$ values ranging from $23.49 \mathrm{mg} / \mathrm{L}$ to $19.69 \mathrm{mg} / \mathrm{L}$ compared to compound $\mathbf{8}$, which delivered information that chlorine atom has the best activity among halogens. Secondly, the typical electron-donating groups $\left(\mathrm{CH}_{3}\right.$ and $\mathrm{CH}_{3} \mathrm{O}$ for compounds 12 and 13 respectively) were imported, we still did not observe any enhancement $\left(\mathrm{EC}_{50}=11.47\right.$ and $14.52 \mathrm{mg} / \mathrm{L}$ respectively $)$, indicating that the electron-donating groups probably have hardly a positive effect on bioactivity. Then we started to conduct the contrary optimization with typical electron-withdrawing groups, compounds 14, 15, 16 were synthesized, respectively. The bioassay results showed that these three compounds showed very good fungicidal activity with much lower $\mathrm{EC}_{50}$ values, especially compound $16\left(\mathrm{EC}_{50}=1.34\right.$ $\mathrm{mg} / \mathrm{L})$, significantly superior to compound $\mathbf{8}$ and even positive controls. The results implied that the groups with strong electron-withdrawing would be likely to play an important role in bioactivity improvement. Enlightened by this result, we further introduced typical stronger electron-withdrawing group $\mathrm{NO}_{2}$ to para-position of phenyl ring. To our excitement, compound $\mathbf{1 7}$ gave a much lower $\mathrm{EC}_{50}$ value of $1.05 \mathrm{mg} / \mathrm{L}$ as anticipated, which was comparable to the commercial fungicide tebuconazole (1.02 $\mathrm{mg} / \mathrm{L})$. However, the introduction of the bulky moiety 
$\mathrm{C}\left(\mathrm{CH}_{3}\right)_{3}$ (compound 18) did not make a greater contribution on bioactivity compared with compound $\mathbf{1 7}$.

Table 2 Fungicidal activity against Rhizoctonia solani of phenylpyrazoloxyl propionic acid derivatives $\mathbf{9} \sim \mathbf{2 0}$

\begin{tabular}{|c|c|c|c|}
\hline Compd. & $\mathrm{R}^{n}$ & $\mathrm{EC}_{50} /\left(\mathrm{mg} \cdot \mathrm{L}^{-1}\right)$ & $95 \% d^{a}$ \\
\hline 9 & $4-\mathrm{F}$ & 20.50 & $13.61 \sim 31.12$ \\
\hline 10 & $4-\mathrm{Br}$ & 19.69 & $15.44 \sim 25.11$ \\
\hline 11 & 4-I & 23.49 & $17.91 \sim 30.82$ \\
\hline 12 & $4-\mathrm{CH}_{3}$ & 11.47 & $8.76 \sim 15.00$ \\
\hline 13 & $4-\mathrm{OCH}_{3}$ & 14.52 & $8.86 \sim 23.81$ \\
\hline 14 & $4-\mathrm{COOCH}_{3}$ & 10.28 & $7.86 \sim 13.44$ \\
\hline 15 & $4-\mathrm{CN}$ & 3.66 & $2.50 \sim 5.33$ \\
\hline 16 & $4-\mathrm{CF}_{3}$ & 1.34 & $0.53 \sim 3.37$ \\
\hline 17 & $4-\mathrm{NO}_{2}$ & 1.05 & $0.45 \sim 2.45$ \\
\hline 18 & $4-\mathrm{C}\left(\mathrm{CH}_{3}\right)_{3}$ & 6.48 & $4.08 \sim 10.26$ \\
\hline 19 & $2,4-\mathrm{Cl}_{2}$ & 7.30 & $5.31 \sim 10.03$ \\
\hline 20 & $3,4-\mathrm{Cl}_{2}$ & 0.95 & $0.26 \sim 2.39$ \\
\hline Tebuconazole & & 1.02 & $0.41 \sim 2.54$ \\
\hline
\end{tabular}

Finally, to investigate the effect of numbers of substituents on fungicidal activity, we started to introduce one more chlorine atom while holding another at para-position, compounds $19\left(\mathrm{R}^{n}=2,4-\mathrm{Cl}_{2}\right)$ and $20\left(\mathrm{R}^{n}=3,4-\mathrm{Cl}_{2}\right)$ were obtained. It is noted that compound 20 possessed a fairly low $\mathrm{EC}_{50}$ of $0.95 \mathrm{mg} / \mathrm{L}$, a little more active than commercial tebuconazole $\left(\mathrm{EC}_{50}=1.02 \mathrm{mg} / \mathrm{L}\right)$.

\subsection{Further development of lead compound}

In order to search widely for a valuable lead, the primary structure modification on $\mathrm{R}^{2}$ of heteroaryl groups was unfolded (Table 3 ). Five compounds $\mathbf{2 1} \sim \mathbf{2 5}$ were obtained by introducing representative pyridinyl or pyridazinyl ring. Among them, compound 22 exhibited an equivalent fungicidal activity to tebuconazole with $\mathrm{EC}_{50}$ of $1.02 \mathrm{mg} / \mathrm{L}$, but it is still slightly inferior to compound $\mathbf{2 0}$.

Table 3 Chemical structures and fungicidal activity against Rhizoctonia solani of phenylpyrazoloxyl propionic acid derivatives $\mathbf{2 1} \sim \mathbf{2 5}$

\begin{tabular}{clcc} 
& & \multicolumn{1}{c}{} \\
\hline Compd. & \multicolumn{1}{c}{$\mathrm{R}^{2}$} & $\mathrm{EC}_{50} /\left(\mathrm{mg}^{\prime} \mathrm{L}^{-1}\right)$ & $95 \% d^{a}$ \\
\hline $\mathbf{2 1}$ & 3-Pyridyl & 1.24 & $0.53 \sim 2.86$ \\
$\mathbf{2 2}$ & 2-Pyridyl & 1.02 & $0.38 \sim 2.70$ \\
$\mathbf{2 3}$ & 3-Methylpyridin-2-yl & 5.95 & $4.42 \sim 8.01$ \\
$\mathbf{2 4}$ & 6-Methylpyridin-2-yl & 4.89 & $3.68 \sim 6.50$ \\
$\mathbf{2 5}$ & 6-Chloropyridazin-3-yl & 10.62 & $8.14 \sim 13.85$ \\
\hline${ }^{a}$ Cofiden
\end{tabular}

${ }^{a}$ Confidence limit.

\section{Conclusions}

In summary, the preliminary bioassay results showed that some of them exhibited excellent fungicidal activity against Rhizoctonia solani, especially compounds 17 $\left(\mathrm{EC}_{50}=1.05 \mathrm{mg} / \mathrm{L}\right)$ and $22\left(\mathrm{EC}_{50}=1.02 \mathrm{mg} / \mathrm{L}\right)$, which showed close activity to commercial contrasts tebuconazole $\left(\mathrm{EC}_{50}=1.02 \mathrm{mg} / \mathrm{L}\right)$, and compound $20\left(\mathrm{EC}_{50}=0.95\right.$ $\mathrm{mg} / \mathrm{L})$ is a little more active than tebuconazole $\left(\mathrm{EC}_{50}=\right.$ $1.02 \mathrm{mg} / \mathrm{L})$. Further structure optimization studies and field trials of compound $\mathbf{2 0}$ are in progress as a promising candidate for further development.

\section{Experimental section}

\subsection{Materials and physical measurements}

All chemicals such as starting materials and reagents were commercially available (Sinopharm Chemical Reagent Co. Ltd., China) and used without further purification except as indicated. Melting points were determined on a M-569 melting point apparatus (Büchi Labortechnik AG, Switzerland) and were uncorrected. ${ }^{1} \mathrm{H}$ NMR and ${ }^{13} \mathrm{C}$ NMR spectra were recorded with a Mercury $300 \mathrm{MHz}$ spectrometer (Varian, Canada) or a Ultra Shield $600 \mathrm{MHz}$ spectrometer (Bruker BioSpin AG, Switzerland) with deuterochloroform $\left(\mathrm{CDCl}_{3}\right)$, dimethyl sulfoxide- $d_{6}\left(\mathrm{DMSO}-d_{6}\right)$ or acetone- $d_{6}\left(\left(\mathrm{CD}_{3}\right)_{2} \mathrm{CO}\right)$ as the solvent and tetramethylsilane (TMS) as the internal standard. Elemental analyses were determined on an elementar Vario EL cube instrument (Elementar Analysensysteme GmbH, Germany). The mass spectra were acquired with an Agilent 1100 series LC/MSD Trap (Agilent Technologies, America) equipped with electron spray ionization (ESI) source. Infrared spectra (IR) were recorded with an Alpha II infrared spectrometer (Bruker, Germany). X-ray structure determination was recorded with D8 Quest single crystal X-ray diffractometer (Bruker AXS Gmbh, Germany). The isolation of the compounds was conducted by Biotage Isolera Prime flash purification system (Biotage, Sweden).

\subsection{Synthetic procedure}

4.2.1 Synthesis of ethyl 2-((1-(4-chlorophenyl)-1Hpyrazol-3-yl)oxy)propanoate (A)

Compound A was prepared according to the previously reported methods. ${ }^{[26]}$ Briefly, the mixture of ethyl 2-bromopropanoate (11.00, $60.0 \mathrm{mmol}), 1$-(4-chlorophenyl)-1H-pyrazol-3-ol $(9.93 \mathrm{~g}, 50.0 \mathrm{mmol})$ and potassium carbonate $(5.60 \mathrm{~g}, 40.0 \mathrm{mmol})$ in $N, N$-dimethylformamide (DMF, $100 \mathrm{~mL}$ ) was stirred at $100{ }^{\circ} \mathrm{C}$ for about $5 \mathrm{~h}$. After completion of the reaction (TLC), the reaction mixture was filtered. The filtrate was evaporated to remove DMF in vacuum and the residue was dissolved in ethyl acetate (50 $\mathrm{mL})$. The organic phase was washed with water $(20 \mathrm{~mL})$, evaporated, and recrystallized from ethanol, then $12.06 \mathrm{~g}$ (81.0\% yield) of $\mathbf{A}$ was obtained as a white solid. m.p. 77 $79{ }^{\circ} \mathrm{C} ;{ }^{1} \mathrm{H}$ NMR $\left(300 \mathrm{MHz}, \mathrm{CDCl}_{3}\right) \delta: 7.69$ (d, $J=2.7$ $\mathrm{Hz}, 1 \mathrm{H}$, pyrazol-5-H), 7.48 (d, $J=8.7 \mathrm{~Hz}, 2 \mathrm{H}, 4-\mathrm{Cl}-\mathrm{Ph}-$ 
2,6-2H), 7.33 (d, $J=8.7 \mathrm{~Hz}, 2 \mathrm{H}, 4-\mathrm{Cl}-\mathrm{Ph}-3,5-2 \mathrm{H}), 5.95$ (d, $J=2.7 \mathrm{~Hz}, 1 \mathrm{H}$, pyrazol-4-H), 5.14 (q, $J=6.9 \mathrm{~Hz}, 1 \mathrm{H}, \mathrm{CH})$, $4.29 \sim 4.19\left(\mathrm{~m}, 2 \mathrm{H}, \mathrm{CH}_{2}\right), 1.63\left(\mathrm{~d}, J=6.9 \mathrm{~Hz}, 3 \mathrm{H}, \mathrm{CH}_{3}\right)$, $1.25\left(\mathrm{t}, J=7.2 \mathrm{~Hz}, 3 \mathrm{H}, \mathrm{CH}_{3}\right) ;{ }^{13} \mathrm{C}$ NMR (DMSO- $d_{6}, 150$ $\mathrm{MHz}) \delta$ : 171.30, 162.70, 138.31, 129.58, 129.26, 129.12, 118.40, 94.41, 72.55, 60.54, 17.77, 14.02; MS m/z: 295.08 $(\mathrm{M}+\mathrm{H})^{+}$. Anal. calcd for $\mathrm{C}_{14} \mathrm{H}_{15} \mathrm{ClN}_{2} \mathrm{O}_{3}$ : C 57.05, H 5.13, $\mathrm{N}$ 9.50; found C 57.00, H 5.12, N 9.52.

\subsubsection{Synthesis of 2-((1-(4-chlorophenyl)-1H-pyrazol-} 3-yl)oxy)propanoic acid (B)

Aqueous solution of $\mathrm{NaOH}(36.00 \mathrm{~g}, 5.0 \%, 45.0 \mathrm{mmol})$ was added dropwise over $5 \mathrm{~m}$ to a stirred mixture of compound $\mathbf{A}(8.93 \mathrm{~g}, 30.0 \mathrm{mmol})$ in ethanol $(99.0 \%, 20 \mathrm{~mL})$, the reaction mixture was stirred at $60{ }^{\circ} \mathrm{C}$ for $2 \mathrm{~h}$. After the reaction completed (TLC), the reaction mixture was extracted with toluene $(99.0 \%, 20 \mathrm{~mL})$ and the organic phase was discarded. The water phase was adjusted to $\mathrm{pH}=3 \sim 4$ with hydrochloric acid $(35.0 \%, w / w)$, and then the resulting mixture was extracted with ethyl acetate $(98.5 \%, 150 \mathrm{~mL})$. After evaporation and re-crystallization treatment (from ethanol), $6.88 \mathrm{~g}$ (85.2\% yield) of $\mathbf{B}$ was obtained as a white solid. m.p. 171 $172{ }^{\circ} \mathrm{C}$; ${ }^{1} \mathrm{H}$ NMR $\left(\left(\mathrm{CD}_{3}\right)_{2} \mathrm{CO}, 300 \mathrm{MHz}\right)$ $\delta: 8.17(\mathrm{~d}, J=2.7 \mathrm{~Hz}, 1 \mathrm{H}$, pyrazol-5-H), $7.72(\mathrm{~d}, J=8.7$ $\mathrm{Hz}, 2 \mathrm{H}, 4-\mathrm{Cl}-\mathrm{Ph}-2,6-2 \mathrm{H}), 7.45$ (d, $J=8.7 \mathrm{~Hz}, 2 \mathrm{H}$, 4-Cl-Ph-3,5-2H), 5.99 (d, $J=2.7 \mathrm{~Hz}, 1 \mathrm{H}$, pyrazol-4-H), $5.16(\mathrm{q}, J=6.9 \mathrm{~Hz}, 1 \mathrm{H}, \mathrm{CH}), 1.61(\mathrm{~d}, J=6.9 \mathrm{~Hz}, 3 \mathrm{H}$, $\left.\mathrm{CH}_{3}\right) ;{ }^{13} \mathrm{C} \mathrm{NMR}\left(\left(\mathrm{CD}_{3}\right)_{2} \mathrm{CO}, 75 \mathrm{MHz}\right) \delta: 173.07,164.21$, $139.64,130.48,130.03,129.48,119.32,95.23,73.15$, 18.32; MS $m / z$ : $267.05(\mathrm{M}+\mathrm{H})^{+}$. Anal. calcd for $\mathrm{C}_{12} \mathrm{H}_{11} \mathrm{ClN}_{2} \mathrm{O}_{3}$ : C 54.05, H 4.16, N 10.50; found C 53.99, H 4.15, N 10.53 .

4.2.3 Synthesis of 2-((1-(4-chlorophenyl)-1H-pyrazol3-yl)oxy)propanoyl chloride (C)

Under ambient condition, intermediate B (0.67 g, $99.1 \%, 2.5 \mathrm{mmol}$ ) was added to sulfurous dichloride (5.00 $\mathrm{g})$. The reaction mixture was heated to reflux and stirred for $3 \mathrm{~h}$. After the excess sulfurous dichloride was removed in vacuum, $0.71 \mathrm{~g}$ of $\mathbf{C}$ was obtained as a brown solid, m.p. $44 \sim 46{ }^{\circ} \mathrm{C}$. It can be used directly for next step without further purification.

4.2.4 General synthetic procedure of compounds $1 \sim$ 25

At room temperature, the substituted amine (D) and intermediate $\mathbf{C}$ in a molar ratio of $1: 1$ were added to dichloromethane. Then the sufficient triethylamine was added cautiously. The reaction mixture was monitored by TLC. After the reaction completed (about $2 \mathrm{~h}$ ), the mixture was washed with water, dried over anhydrous sodium sulfate and evaporated. The crude product was purified by Biotage flash purification system using mixture of ethyl acetate and petroleum ether (boiling point range $60 \sim$ $90{ }^{\circ} \mathrm{C}$ ) as the eluent to obtain corresponding compounds. The yields were not optimized, and each new compound was identified and verified by ${ }^{1} \mathrm{H}$ NMR, ${ }^{13} \mathrm{C}$ NMR, IR, MS and elemental analyses.

\subsection{Spectral data of compounds $1 \sim 25$}

2-((1-(4-Chlorophenyl)-1H-pyrazol-3-yl)oxy)- $N, N$-diethylpropanamide (1): 77\% yield. White solid, m.p. 117 $119{ }^{\circ} \mathrm{C} ;{ }^{1} \mathrm{H} \mathrm{NMR}\left(\mathrm{CDCl}_{3}, 300 \mathrm{MHz}\right) \delta: 7.67(\mathrm{~d}, J=2.7 \mathrm{~Hz}$, $1 \mathrm{H}$, pyrazol-5-H), 7.46 (d, $J=9.0 \mathrm{~Hz}, 2 \mathrm{H}, 4-\mathrm{Cl}-\mathrm{Ph}-2,6-$ 2H), 7.34 (d, $J=9.0 \mathrm{~Hz}, 2 \mathrm{H}, 4-\mathrm{Cl}-\mathrm{Ph}-3,5-2 \mathrm{H}), 5.96$ (d, $J=$ $2.7 \mathrm{~Hz}, 1 \mathrm{H}$, pyrazol-4-H), 5.40 (q, $J=6.9 \mathrm{~Hz}, 1 \mathrm{H}, \mathrm{CH}$ ), $3.63 \sim 3.32\left(\mathrm{~m}, 4 \mathrm{H}, 2 \mathrm{CH}_{2}\right), 1.60\left(\mathrm{~d}, J=6.9 \mathrm{~Hz}, 3 \mathrm{H}, \mathrm{CH}_{3}\right)$, $1.30\left(\mathrm{t}, J=6.9 \mathrm{~Hz}, 3 \mathrm{H}, \mathrm{CH}_{3}\right), 1.12\left(\mathrm{t}, J=6.9 \mathrm{~Hz}, 3 \mathrm{H}, \mathrm{CH}_{3}\right)$; ${ }^{13} \mathrm{C}$ NMR (DMSO- $\left.d_{6}, 150 \mathrm{MHz}\right) \delta: 169.44,162.80$, $138.42,129.53,129.26,128.92,118.36,94.66,70.55$, $40.99,39.77,18.09,14.33,12.79$; $\mathrm{MS} \mathrm{m} / z$ : $322.2(\mathrm{M}+$ $\mathrm{H})^{+}$. Anal. calcd for $\mathrm{C}_{16} \mathrm{H}_{20} \mathrm{ClN}_{3} \mathrm{O}_{2}$ : C 59.72, $\mathrm{H}$ 6.26, N 13.06; found C 59.62, H 6.25, N 13.08.

$N$-Butyl-2-((1-(4-chlorophenyl)-1H-pyrazol-3-yl)oxy)propanamide (2): 79\% yield. Pale yellow oil. ${ }^{1} \mathrm{H}$ NMR $\left(\mathrm{CDCl}_{3}, 300 \mathrm{MHz}\right) \delta: 7.74(\mathrm{~d}, J=2.7 \mathrm{~Hz}, 1 \mathrm{H}$, pyrazol-5H), 7.52 (d, $J=9.0 \mathrm{~Hz}, 2 \mathrm{H}, 4-\mathrm{Cl}-\mathrm{Ph}-2,6-2 \mathrm{H}), 7.36$ (d, $J=$ $9.0 \mathrm{~Hz}, 2 \mathrm{H}, 4-\mathrm{Cl}-\mathrm{Ph}-3,5-2 \mathrm{H}), 6.49$ (s, 1H, NH), 5.95 (d, $J=2.7 \mathrm{~Hz}, 1 \mathrm{H}$, pyrazol-4-H), 5.09 (q, $J=6.9 \mathrm{~Hz}, 1 \mathrm{H}, \mathrm{CH})$, $3.32 \sim 3.26\left(\mathrm{~m}, 2 \mathrm{H}, \mathrm{CH}_{2}\right), 1.62\left(\mathrm{~d}, J=6.9 \mathrm{~Hz}, 3 \mathrm{H}, \mathrm{CH}_{3}\right)$, $1.50 \sim 1.42\left(\mathrm{~m}, 2 \mathrm{H}, \mathrm{CH}_{2}\right), 1.32 \sim 1.25\left(\mathrm{~m}, 2 \mathrm{H}, \mathrm{CH}_{2}\right), 0.85$ $\left(\mathrm{t}, J=6.9 \mathrm{~Hz}, 3 \mathrm{H}, \mathrm{CH}_{3}\right) ;{ }^{13} \mathrm{C} \mathrm{NMR}$ (DMSO- $\left.d_{6}, 150 \mathrm{MHz}\right)$ $\delta: 170.57,162.83,138.40,129.30,129.23,129.02,118.51$, 94.75, 74.52, 37.88, 31.19, 19.34, 18.48, 13.59; MS m/z: $322.1(\mathrm{M}+\mathrm{H})^{+}$. Anal. calcd for $\mathrm{C}_{16} \mathrm{H}_{20} \mathrm{ClN}_{3} \mathrm{O}_{2}: \mathrm{C} 59.72, \mathrm{H}$ 6.26, N 13.06; found C 59.68, H 6.25, N 13.09.

2-((1-(4-Chlorophenyl)-1H-pyrazol-3-yl)oxy)- $N$-cyclopropylpropanamide (3): $73 \%$ yield. White solid, m.p. $141 \sim 143{ }^{\circ} \mathrm{C} ;{ }^{1} \mathrm{H}$ NMR $\left(\mathrm{CDCl}_{3}, 300 \mathrm{MHz}\right) \delta: 7.73$ (d, $J=$ $2.7 \mathrm{~Hz}, 1 \mathrm{H}$, pyrazol-5-H), 7.52 (d, $J=9.3 \mathrm{~Hz}, 2 \mathrm{H}, 4-\mathrm{Cl}-$ Ph-2,6-2H), 7.38 (d, $J=9.3 \mathrm{~Hz}, 2 \mathrm{H}, 4-\mathrm{Cl}-\mathrm{Ph}-3,5-2 \mathrm{H}), 6.54$ (s, 1H, NH), 5.93 (d, $J=2.7 \mathrm{~Hz}, 1 \mathrm{H}$, pyrazol-4-H), 5.07 (q, $J=6.9 \mathrm{~Hz}, 1 \mathrm{H}, \mathrm{CH}), 2.77 \sim 2.71(\mathrm{~m}, 1 \mathrm{H}, \mathrm{CH}), 1.60(\mathrm{~d}, J=$ $\left.6.9 \mathrm{~Hz}, 3 \mathrm{H}, \mathrm{CH}_{3}\right), 0.79 \sim 0.76\left(\mathrm{~m}, 2 \mathrm{H}, \mathrm{CH}_{2}\right), 0.51 \sim 0.49$ $\left(\mathrm{m}, 2 \mathrm{H}, \mathrm{CH}_{2}\right) ;{ }^{13} \mathrm{C}$ NMR (DMSO- $\left.d_{6}, 150 \mathrm{MHz}\right) \delta: 171.86$, $162.79,138.40,129.33,129.28,129.03,118.46,94.73$, 74.23, 22.19, 18.28, 5.77, 5.46; MS m/z: $306.1(\mathrm{M}+\mathrm{H})^{+}$. Anal. calcd for $\mathrm{C}_{15} \mathrm{H}_{16} \mathrm{ClN}_{3} \mathrm{O}_{2}$ : C 58.92, H 5.27, N 13.74; found $\mathrm{C} 58.86, \mathrm{H} 5.25, \mathrm{~N} 13.77$.

2-((1-(4-Chlorophenyl)-1H-pyrazol-3-yl)oxy)- $N$-cyclopentylpropanamide (4): $70 \%$ yield. Pale yellow oil. ${ }^{1} \mathrm{H}$ NMR $\left(\mathrm{CDCl}_{3}, 300 \mathrm{MHz}\right) \delta: 7.74(\mathrm{~d}, J=2.7 \mathrm{~Hz}, 1 \mathrm{H}$, pyrazol-5-H), 7.53 (d, $J=8.7 \mathrm{~Hz}, 2 \mathrm{H}, 4-\mathrm{Cl}-\mathrm{Ph}-2,6-2 \mathrm{H})$, 7.37 (d, $J=8.7 \mathrm{~Hz}, 2 \mathrm{H}, 4-\mathrm{Cl}-\mathrm{Ph}-3,5-2 \mathrm{H}), 6.39$ (s, 1H, NH), 5.95 (d, $J=2.7 \mathrm{~Hz}, 1 \mathrm{H}$, pyrazol-4-H), 5.04 (q, $J=7.2 \mathrm{~Hz}$, $1 \mathrm{H}, \mathrm{CH}), 4.27 \sim 4.20(\mathrm{~m}, 1 \mathrm{H}, \mathrm{CH}), 2.02 \sim 1.90(\mathrm{~m}, 2 \mathrm{H}$, $\left.\mathrm{CH}_{2}\right), 1.62 \sim 1.59\left(\mathrm{~m}, 7 \mathrm{H}, \mathrm{CH}_{3} ; 2 \mathrm{CH}_{2}\right), 1.53 \sim 1.37(\mathrm{~m}, 2 \mathrm{H}$, $\left.\mathrm{CH}_{2}\right) ;{ }^{13} \mathrm{C}$ NMR (DMSO- $\left.d_{6}, 150 \mathrm{MHz}\right) \delta: 170.12,162.84$, $138.41,129.26,129.23,129.00,118.43,94.69,74.26$, $50.05,32.22,32.01,23.49,23.47,18.37$; MS m/z: 334.2 $(\mathrm{M}+\mathrm{H})^{+}$. Anal. calcd for $\mathrm{C}_{17} \mathrm{H}_{20} \mathrm{ClN}_{3} \mathrm{O}_{2}$ : C 61.17, $\mathrm{H}$ 6.04, $\mathrm{N}$ 12.59; found C 61.11, H 6.02, N 12.63.

2-((1-(4-Chlorophenyl)-1H-pyrazol-3-yl)oxy)- $N$-phenylpropanamide (5): $88 \%$ yield. White solid, m.p. 154 $156{ }^{\circ} \mathrm{C} ;{ }^{1} \mathrm{H} \mathrm{NMR}\left(\mathrm{CDCl}_{3}, 300 \mathrm{MHz}\right) \delta: 8.25$ (s, $\left.1 \mathrm{H}, \mathrm{NH}\right)$, 
$7.75(\mathrm{~d}, J=2.7 \mathrm{~Hz}, 1 \mathrm{H}$, pyrazol-5-H), $7.58 \sim 7.52(\mathrm{~m}, 4 \mathrm{H}$, 4-Cl-Ph-2,6-2H; Ph-2,6-2H), 7.39 7.36 (m, 4H; 4-Cl-Ph3,5-2H; Ph-3,5-2H), 7.33 (t, $J=7.2 \mathrm{~Hz}, 1 \mathrm{H}, \mathrm{Ph}-4-\mathrm{H}), 6.02$ $(\mathrm{d}, J=2.7 \mathrm{~Hz}, 1 \mathrm{H}$; pyrazol-4-H), 5.24 (q, $J=6.9 \mathrm{~Hz}, 1 \mathrm{H}$, $\mathrm{CH}), 1.72\left(\mathrm{~d}, J=6.9 \mathrm{~Hz}, 3 \mathrm{H}, \mathrm{CH}_{3}\right) ;{ }^{13} \mathrm{C}$ NMR (DMSO- $d_{6}$, $150 \mathrm{MHz}) \delta$ : 169.77, 162.79, 138.66, 138.35, 129.52, $129.26,129.06,128.69,123.51,119.57,118.45$, 94.70, 74.51, 18.32; MS m/z: $342.2(\mathrm{M}+\mathrm{H})^{+}$. Anal. calcd for $\mathrm{C}_{18} \mathrm{H}_{16} \mathrm{ClN}_{3} \mathrm{O}_{2}$ : C 63.25, $\mathrm{H}$ 4.72, N 12.29; found $\mathrm{C}$ 63.23, $\mathrm{H}$ 4.71, N 12.30.

$\mathrm{N}$-(2-Chlorophenyl)-2-((1-(4-chlorophenyl)-1H-pyrazol3-yl)oxy)propanamide (6): $87 \%$ yield. White solid, m.p. 98 $100{ }^{\circ} \mathrm{C} ;{ }^{1} \mathrm{H}$ NMR $\left(\mathrm{CDCl}_{3}, 300 \mathrm{MHz}\right) \delta: 8.85(\mathrm{~s}, 1 \mathrm{H}$, $\mathrm{NH}), 8.43$ (dd, $\left.{ }^{3} J=8.1 \mathrm{~Hz},{ }^{4} J=1.5 \mathrm{~Hz}, 1 \mathrm{H}, 2-\mathrm{Cl}-\mathrm{Ph}-6-\mathrm{H}\right)$, 7.74 (d, $J=2.4 \mathrm{~Hz}, 1 \mathrm{H}$, pyrazol-5-H), 7.53 (d, $J=9.0 \mathrm{~Hz}$, $2 \mathrm{H}, 4-\mathrm{Cl}-\mathrm{Ph}-2,6-2 \mathrm{H}$ ), $7.38 \sim 7.25$ (m, 4H, 4-Cl-Ph-3,5-2H; 2-Cl-Ph-3,5-2H), 7.03 (ddd, ${ }^{3} J_{1}=7.8,7.8 \mathrm{~Hz},{ }^{4} J=1.5 \mathrm{~Hz}$, $1 \mathrm{H}, 2-\mathrm{Cl}-\mathrm{Ph}-4-\mathrm{H}), 6.03$ (d, $J=2.4 \mathrm{~Hz}, 1 \mathrm{H}$, pyrazol-4-H), $5.36(\mathrm{q}, J=6.9 \mathrm{~Hz}, 1 \mathrm{H}, \mathrm{CH}), 1.74(\mathrm{~d}, J=6.9 \mathrm{~Hz}, 3 \mathrm{H}$, $\left.\mathrm{CH}_{3}\right) ;{ }^{13} \mathrm{C}$ NMR (DMSO- $\left.d_{6}, 150 \mathrm{MHz}\right) \delta: 170.10,162.68$, $138.33,134.29,129.53,129.45,129.22,129.13,127.47$, $126.60,126.48,125.82,118.51,94.69,74.33,18.36$; MS $m / z$ : $376.1(\mathrm{M}+\mathrm{H})^{+}$. Anal. calcd for $\mathrm{C}_{18} \mathrm{H}_{15} \mathrm{Cl}_{2} \mathrm{~N}_{3} \mathrm{O}_{2}$ : C 57.46, H 4.02, N 11.17; found C 57.39, H 4.01, N 11.20.

$\mathrm{N}$-(3-Chlorophenyl)-2-((1-(4-chlorophenyl)-1H-pyrazol3-yl)oxy)propanamide (7): $84 \%$ yield. White solid, m.p. $117 \sim 119{ }^{\circ} \mathrm{C} ;{ }^{1} \mathrm{H} \mathrm{NMR}\left(\mathrm{CDCl}_{3}, 300 \mathrm{MHz}\right) \delta: 8.28(\mathrm{~s}, 1 \mathrm{H}$, $\mathrm{NH}), 7.75(\mathrm{~d}, J=2.7 \mathrm{~Hz}, 1 \mathrm{H}$, pyrazol-5-H), 7.70 (dd, $1 \mathrm{H}$, $\left.{ }^{4} J_{1}=1.8,2.1 \mathrm{~Hz}, 3-\mathrm{Cl}-\mathrm{Ph}-2-\mathrm{H}\right), 7.53(\mathrm{~d}, J=8.7 \mathrm{~Hz}, 2 \mathrm{H}$, 4-Cl-Ph-2,6-2H), $7.42 \sim 7.36$ (m, 3H, 4-Cl-Ph-3,5-2H; 3-Cl-Ph-6-H), 7.24 (dd, ${ }^{3} J=8.1,8.1 \mathrm{~Hz}, 1 \mathrm{H}, 3-\mathrm{Cl}-\mathrm{Ph}-5-\mathrm{H}$ ), 7.09 (ddd, ${ }^{3} \mathrm{~J}=8.1 \mathrm{~Hz},{ }^{4} \mathrm{~J}=2.1,0.9 \mathrm{~Hz}, 1 \mathrm{H}, 3-\mathrm{Cl}-\mathrm{Ph}-4-\mathrm{H}$ ), $6.01(\mathrm{~d}, J=2.7 \mathrm{~Hz}, 1 \mathrm{H}$, pyrazol-4-H), 5.24 (q, $J=6.9 \mathrm{~Hz}$, $1 \mathrm{H}, \mathrm{CH}), 1.71\left(\mathrm{~d}, J=6.9 \mathrm{~Hz}, 3 \mathrm{H}, \mathrm{CH}_{3}\right) ;{ }^{13} \mathrm{C} \mathrm{NMR}$ (DMSO- $\left.d_{6}, 150 \mathrm{MHz}\right) \delta: 170.23,162.71,140.10,138.32$, $133.01,130.44,129.59,129.27,129.10,123.25,119.02$, $118.45,117.95,94.69,74.49,18.23$; MS m/z: $376.1(\mathrm{M}+$ $\mathrm{H})^{+}$; Anal. calcd for $\mathrm{C}_{18} \mathrm{H}_{15} \mathrm{Cl}_{2} \mathrm{~N}_{3} \mathrm{O}_{2}$ : C 57.46, $\mathrm{H} 4.02, \mathrm{~N}$ 11.17; found C 57.41, $\mathrm{H} 4.01, \mathrm{~N} 11.20$.

$\mathrm{N}$-(4-Chlorophenyl)-2-((1-(4-chlorophenyl)-1H-pyrazol3-yl)oxy)propanamide (8): $86 \%$ yield. White solid, m.p. $159 \sim 161{ }^{\circ} \mathrm{C} ;{ }^{1} \mathrm{H}$ NMR $\left(\mathrm{CDCl}_{3}, 600 \mathrm{MHz}\right) \delta: 8.29$ (s, $1 \mathrm{H}$, $\mathrm{NH}), 7.74(\mathrm{~d}, J=2.7 \mathrm{~Hz}, 1 \mathrm{H}$, pyrazol-5-H), $7.53 \sim 7.51(\mathrm{~m}$, 4H, 4-Cl-Ph-2,6-2H; 4-Cl-Ph-2,6-2H), 7.37 (d, $J=8.7 \mathrm{~Hz}$, 2H, 4-Cl-Ph-3,5-2H), 7.28 (d, $J=8.7 \mathrm{~Hz}, 2 \mathrm{H}, 4-\mathrm{Cl}-\mathrm{Ph}-$ $3,5-2 \mathrm{H}), 6.00(\mathrm{~d}, J=2.7 \mathrm{~Hz}, 1 \mathrm{H}$, pyrazol-4-H), 5.23 (q, $J=$ $6.9 \mathrm{~Hz}, 1 \mathrm{H}, \mathrm{CH}), 1.70\left(\mathrm{~d}, J=6.9 \mathrm{~Hz}, 3 \mathrm{H}, \mathrm{CH}_{3}\right) ;{ }^{13} \mathrm{C} \mathrm{NMR}$ $\left(\mathrm{CDCl}_{3}, 150 \mathrm{MHz}\right) \delta: 169.88,162.59,138.39,135.92$, $131.31,129.68,129.58,129.13,128.23,121.33,119.11$, 94.65, 76.23, 18.37; MS m/z: $376.1(\mathrm{M}+\mathrm{H})^{+}$. Anal. calcd

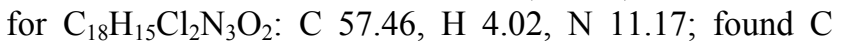
57.40, H 4.01, N 11.19.

2-((1-(4-Chlorophenyl)-1H-pyrazol-3-yl)oxy)- $N$-(4-fluorophenyl)propanamide (9): $83 \%$ yield. White solid, m.p. $146 \sim 148{ }^{\circ} \mathrm{C} ;{ }^{1} \mathrm{H}$ NMR $\left(\mathrm{CDCl}_{3}, 300 \mathrm{MHz}\right) \delta: 8.24(\mathrm{~s}, 1 \mathrm{H}$, $\mathrm{NH}), 7.76(\mathrm{~d}, J=2.4 \mathrm{~Hz}, 1 \mathrm{H}$, pyrazol-5-H), $7.55 \sim 7.50(\mathrm{~m}$, 4H, 4-Cl-Ph-2,6-2H; 4-F-Ph-2,6-2H), 7.40 (d, $J=9.0 \mathrm{~Hz}$,
2H, 4-Cl-Ph-3,5-2H), 7.02 (t, $J=8.7 \mathrm{~Hz}, 2 \mathrm{H}, 4-\mathrm{F}-\mathrm{Ph}-$ $3,5-2 \mathrm{H}), 6.01(\mathrm{~d}, J=2.4 \mathrm{~Hz}, 1 \mathrm{H}$, pyrazol-4-H), 5.24 (q, $J=$ $6.6 \mathrm{~Hz}, 1 \mathrm{H}, \mathrm{CH}), 1.71\left(\mathrm{~d}, J=6.6 \mathrm{~Hz}, 3 \mathrm{H}, \mathrm{CH}_{3}\right) ;{ }^{13} \mathrm{C} \mathrm{NMR}$ $\left(\mathrm{DMSO}-d_{6}, 150 \mathrm{MHz}\right) \delta$ : $169.72,162.76,158.14\left(\mathrm{~d},{ }^{1} J=\right.$ $238.50 \mathrm{~Hz}, \mathrm{~F}-\mathrm{C}), 138.34,135.02$ (d, $\left.{ }^{4} J=2.25 \mathrm{~Hz}, \mathrm{~F}-\mathrm{C}\right)$, $129.53,129.27,129.08,121.39$ (d, $\left.{ }^{3} J=7.65 \mathrm{~Hz}, \mathrm{~F}-\mathrm{C}\right)$, $118.45,115.26\left(\mathrm{~d},{ }^{2} J=22.50 \mathrm{~Hz}, \mathrm{~F}-\mathrm{C}\right), 94.70,74.53$, 18.30. MS $m / z: 360.1(\mathrm{M}+\mathrm{H})^{+}$. Anal. calcd for $\mathrm{C}_{18} \mathrm{H}_{15^{-}}$ $\mathrm{ClFN}_{3} \mathrm{O}_{2}$ : C 60.09, $\mathrm{H}$ 4.20, N 11.68; found $\mathrm{C} 60.03, \mathrm{H}$ 4.20, N 11.70.

$\mathrm{N}$-(4-Bromophenyl)-2-((1-(4-chlorophenyl)-1H-pyrazol3-yl)oxy)propanamide (10): $80 \%$ yield. White solid, m.p. $157 \sim 159{ }^{\circ} \mathrm{C} ;{ }^{1} \mathrm{H}$ NMR $\left(\mathrm{CDCl}_{3}, 300 \mathrm{MHz}\right) \delta: 8.28(\mathrm{~s}, 1 \mathrm{H}$, $\mathrm{NH}), 7.75(\mathrm{~d}, J=2.7 \mathrm{~Hz}, 1 \mathrm{H}$, pyrazol-5-H), $7.52(\mathrm{~d}, J=9.0$ $\mathrm{Hz}, 2 \mathrm{H}, 4-\mathrm{Cl}-\mathrm{Ph}-2,6-2 \mathrm{H}$ ), 7.45 (m, 4H, 4-Br-Ph-2,6-2H; 4-Br-Ph-3,5-2H), 7.37 (d, $J=9.0 \mathrm{~Hz}, 2 \mathrm{H}, 4-\mathrm{Cl}-\mathrm{Ph}-3,5-2 \mathrm{H})$, $6.01(\mathrm{~d}, J=2.7 \mathrm{~Hz}, 1 \mathrm{H}$, pyrazol-4-H), 5.24 (q, $J=6.9 \mathrm{~Hz}$, $1 \mathrm{H}, \mathrm{CH}), 1.70\left(\mathrm{~d}, J=6.9 \mathrm{~Hz}, 3 \mathrm{H}, \mathrm{CH}_{3}\right) ;{ }^{13} \mathrm{C} \mathrm{NMR}$ (DMSO- $\left.d_{6}, 150 \mathrm{MHz}\right) \delta: 169.98,162.72,138.32,138.02$, $131.52,129.56,129.28,129.09$, 121.51, 118.44, 115.16, 94.69, 74.51, 18.24; MS m/z: $420.0(\mathrm{M}+\mathrm{H})^{+}$. Anal. calcd for $\mathrm{C}_{18} \mathrm{H}_{15} \mathrm{BrClN}_{3} \mathrm{O}_{2}$ : C 51.39, H 3.59, N 9.99; found $\mathrm{C}$ 51.34, H 3.58, N 10.01.

2-((1-(4-Chlorophenyl)-1H-pyrazol-3-yl)oxy)- $N$-(4-iodophenyl)propanamide (11): $84 \%$ yield. White solid, m.p. $162 \sim 164{ }^{\circ} \mathrm{C} ;{ }^{1} \mathrm{H}$ NMR $\left(\mathrm{CDCl}_{3}, 300 \mathrm{MHz}\right) \delta: 8.26(\mathrm{~s}, 1 \mathrm{H}$, $\mathrm{NH}), 7.75(\mathrm{~d}, J=2.7 \mathrm{~Hz}, 1 \mathrm{H}$, pyrazol-5-H), 7.62 (d, $J=9.0$ $\mathrm{Hz}, 2 \mathrm{H}, 4-\mathrm{I}-\mathrm{Ph}-3,5-2 \mathrm{H}), 7.52$ (d, $J=9.0 \mathrm{~Hz}, 2 \mathrm{H}, 4-\mathrm{Cl}-$ $\mathrm{Ph}-2,6-2 \mathrm{H}), 7.38$ (d, $J=9.0 \mathrm{~Hz}, 2 \mathrm{H}, 4-\mathrm{Cl}-\mathrm{Ph}-3,5-2 \mathrm{H}), 7.36$ (d, $J=9.0 \mathrm{~Hz}, 2 \mathrm{H}, 4-\mathrm{I}-\mathrm{Ph}-2,6-2 \mathrm{H}), 6.01$ (d, $J=2.7 \mathrm{~Hz}, 1 \mathrm{H}$, pyrazol-4-H), 5.23 (q, $J=6.6 \mathrm{~Hz}, 1 \mathrm{H}, \mathrm{CH}), 1.70$ (d, $J=6.6$ $\left.\mathrm{Hz}, 3 \mathrm{H}, \mathrm{CH}_{3}\right) ;{ }^{13} \mathrm{C}$ NMR (DMSO- $\left.d_{6}, 150 \mathrm{MHz}\right) \delta: 169.97$, $162.72,138.49,138.32,137.35,129.56,129.28,129.08$, $121.77,118.43,94.68,87.11,74.52,18.24$; MS $m / z: 468.0$ $(\mathrm{M}+\mathrm{H})^{+}$. Anal. calcd for $\mathrm{C}_{18} \mathrm{H}_{15} \mathrm{ClIN}_{3} \mathrm{O}_{2}: \mathrm{C} 46.23, \mathrm{H} 3.23$, $\mathrm{N}$ 8.98; found 46.19, H 3.22, N 9.00.

2-((1-(4-Chlorophenyl)-1H-pyrazol-3-yl)oxy)- $N$-( $p$-tolyl)propanamide (12): $88 \%$ yield. White solid, m.p. 132 $134{ }^{\circ} \mathrm{C} ;{ }^{1} \mathrm{H}$ NMR $\left(\mathrm{CDCl}_{3}, 300 \mathrm{MHz}\right) \delta: 8.18(\mathrm{~s}, 1 \mathrm{H}, \mathrm{NH})$, $7.74(\mathrm{~d}, J=2.7 \mathrm{~Hz}, 1 \mathrm{H}$, pyrazol-5-H), 7.53 (d, $J=8.7 \mathrm{~Hz}$, 2H, 4-Cl-Ph-2,6-2H), 7.44 (d, $J=8.4 \mathrm{~Hz}, 2 \mathrm{H}, 4-\mathrm{CH}_{3}-\mathrm{Ph}-$ 2,6-2H), 7.39 (d, $J=8.7 \mathrm{~Hz}, 2 \mathrm{H}, 4-\mathrm{Cl}-\mathrm{Ph}-3,5-2 \mathrm{H}), 7.19$ (d, $\left.J=8.4 \mathrm{~Hz}, 2 \mathrm{H}, 4-\mathrm{CH}_{3}-\mathrm{Ph}-3,5-2 \mathrm{H}\right), 6.00(\mathrm{~d}, J=2.7 \mathrm{~Hz}, 1 \mathrm{H}$, pyrazol-4-H), 5.22 (q, $J=6.9 \mathrm{~Hz}, 1 \mathrm{H}, \mathrm{CH}), 2.30(\mathrm{~s}, 3 \mathrm{H}$, $\left.\mathrm{CH}_{3}\right), 1.71\left(\mathrm{~d}, J=6.9 \mathrm{~Hz}, 3 \mathrm{H}, \mathrm{CH}_{3}\right) ;{ }^{13} \mathrm{C}$ NMR (DMSO- $d_{6}$, $150 \mathrm{MHz}) \delta$ : 169.52, 162.80, 138.36, 136.14, 132.44, $129.46,129.24,129.06,129.04,119.62,118.45,94.70$, 74.53, 20.42, 18.33; MS $m / z: 356.1(\mathrm{M}+\mathrm{H})^{+}$. Anal. calcd

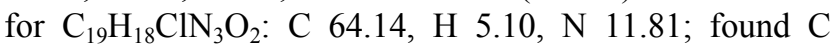
64.10, H 5.09, N 11.84 .

2-((1-(4-Chlorophenyl)-1H-pyrazol-3-yl)oxy)- $N$-(4-methoxyphenyl)propanamide (13): 79\% yield. White solid, m.p. $145 \sim 147{ }^{\circ} \mathrm{C} ;{ }^{1} \mathrm{H}$ NMR $\left(\mathrm{CDCl}_{3}, 300 \mathrm{MHz}\right) \delta: 8.15$ (s, $1 \mathrm{H}, \mathrm{NH}), 7.75$ (d, $J=2.7 \mathrm{~Hz}, 1 \mathrm{H}$, pyrazol-5-H), 7.54 (d, $J=9.0 \mathrm{~Hz}, 2 \mathrm{H}, 4-\mathrm{Cl}-\mathrm{Ph}-2,6-2 \mathrm{H}), 7.46$ (d, $J=9.0 \mathrm{~Hz}, 2 \mathrm{H}$, 4- $\left.\mathrm{CH}_{3} \mathrm{O}-\mathrm{Ph}-2,6-2 \mathrm{H}\right), 7.38$ (d, $J=9.0 \mathrm{~Hz}, 2 \mathrm{H}, 4-\mathrm{Cl}-\mathrm{Ph}-3,5-$ 2H), 6.87 (d, $\left.J=9.0 \mathrm{~Hz}, 2 \mathrm{H}, 4-\mathrm{CH}_{3} \mathrm{O}-\mathrm{Ph}-3,5-2 \mathrm{H}\right), 6.01$ (d, 
$J=2.7 \mathrm{~Hz}, 1 \mathrm{H}$, pyrazol-4-H), 5.23 (q, $J=6.9 \mathrm{~Hz}, 1 \mathrm{H}, \mathrm{CH})$, $3.79\left(\mathrm{~s}, 3 \mathrm{H}, \mathrm{CH}_{3}\right), 1.71\left(\mathrm{~d}, J=6.9 \mathrm{~Hz}, 3 \mathrm{H}, \mathrm{CH}_{3}\right) ;{ }^{13} \mathrm{C} \mathrm{NMR}$ (DMSO- $\left.d_{6}, 150 \mathrm{MHz}\right) \delta: 169.24,162.80,155.40,138.37$, 131.72 , 129.48, 129.27, 129.05, 121.16, 118.46, 113.80, 94.72, 74.5, 55.15, 18.34; MS m/z: $372.2(\mathrm{M}+\mathrm{H})^{+}$. Anal. calcd for $\mathrm{C}_{19} \mathrm{H}_{18} \mathrm{ClN}_{3} \mathrm{O}_{3}$ : C 61.38, H 4.88, N 11.30; found C 61.33, H 4.88, N 11.32.

Methyl 4-(2-((1-(4-chlorophenyl)-1H-pyrazol-3-yl)oxy)propanamido)benzoate (14): $85 \%$ yield. White solid, m.p. $130 \sim 132{ }^{\circ} \mathrm{C} ;{ }^{1} \mathrm{H}$ NMR $\left(\mathrm{CDCl}_{3}, 300 \mathrm{MHz}\right) \delta: 8.43(\mathrm{~s}, 1 \mathrm{H}$, $\mathrm{NH}), 8.02$ (d, $\left.J=8.7 \mathrm{~Hz}, 2 \mathrm{H}, 4-\mathrm{CH}_{3} \mathrm{OOC}-\mathrm{Ph}-3,5-2 \mathrm{H}\right), 7.76$ (d, $J=2.4 \mathrm{~Hz}, 1 \mathrm{H}$, pyrazol-5-H), $7.66(\mathrm{~d}, J=8.7 \mathrm{~Hz}, 2 \mathrm{H}$, 4-CH $\mathrm{CHOC}_{3} \mathrm{OH}-2,6-2 \mathrm{H}$ ), 7.53 (d, $J=8.7 \mathrm{~Hz}, 2 \mathrm{H}, 4-\mathrm{Cl}-\mathrm{Ph}-$ 2,6-2H), 7.38 (d, $J=8.7 \mathrm{~Hz}, 2 \mathrm{H}, 4-\mathrm{Cl}-\mathrm{Ph}-3,5-2 \mathrm{H}), 6.02$ (d, $J=2.4 \mathrm{~Hz}, 1 \mathrm{H}$, pyrazol-4-H), 5.27 (q, $J=6.9 \mathrm{~Hz}, 1 \mathrm{H}, \mathrm{CH})$, $3.90\left(\mathrm{~s}, 3 \mathrm{H}, \mathrm{CH}_{3}\right), 1.72\left(\mathrm{~d}, J=6.9 \mathrm{~Hz}, 3 \mathrm{H}, \mathrm{CH}_{3}\right) ;{ }^{13} \mathrm{C} \mathrm{NMR}$ (DMSO- $\left.d_{6}, 150 \mathrm{MHz}\right) \delta: 170.44,165.78,162.71,143.08$, $138.31,130.25,129.61,129.28,129.09,124.24,118.96$, $118.43,94.67,74.46,51.90,18.23$; MS m/z: $400.2(\mathrm{M}+$ $\mathrm{H})^{+}$. Anal. calcd for $\mathrm{C}_{20} \mathrm{H}_{18} \mathrm{ClN}_{3} \mathrm{O}_{4}: \mathrm{C} 60.08, \mathrm{H} 4.54, \mathrm{~N}$ 10.51; found $\mathrm{C} 60.05, \mathrm{H} 4.53, \mathrm{~N} 10.52$.

2-((1-(4-Chlorophenyl)-1H-pyrazol-3-yl)oxy)- $N$-(4-cyanophenyl)propanamide (15): $81 \%$ yield. White solid, m.p. 155 $157{ }^{\circ} \mathrm{C} ;{ }^{1} \mathrm{H}$ NMR $\left(\mathrm{CDCl}_{3}, 300 \mathrm{MHz}\right) \delta: 8.51(\mathrm{~s}, 1 \mathrm{H}$, $\mathrm{NH}), 7.76(\mathrm{~d}, J=2.7 \mathrm{~Hz}, 1 \mathrm{H}$, pyrazol-5-H), 7.72 (d, $J=9.0$ $\mathrm{Hz}, 2 \mathrm{H}, 4-\mathrm{CN}-\mathrm{Ph}-2,6-2 \mathrm{H}), 7.52$ (d, $J=9.0 \mathrm{~Hz}, 2 \mathrm{H}, 4-\mathrm{Cl}-$ $\mathrm{Ph}-2,6-2 \mathrm{H}), 7.45$ (d, $J=9.0 \mathrm{~Hz}, 2 \mathrm{H}, 4-\mathrm{CN}-\mathrm{Ph}-3,5-2 \mathrm{H})$, 7.38 (d, $J=9.0 \mathrm{~Hz}, 2 \mathrm{H}, 4-\mathrm{Cl}-\mathrm{Ph}-3,5-2 \mathrm{H}), 6.02$ (d, $J=2.7$ $\mathrm{Hz}, 1 \mathrm{H}$, pyrazol-4-H), 5.27 (q, $J=6.9 \mathrm{~Hz}, 1 \mathrm{H}, \mathrm{CH}), 1.71$ $\left(\mathrm{d}, J=6.9 \mathrm{~Hz}, 3 \mathrm{H}, \mathrm{CH}_{3}\right) ;{ }^{13} \mathrm{C}$ NMR (DMSO- $\left.d_{6}, 150 \mathrm{MHz}\right)$ $\delta: 170.69,162.67,142.90,138.29,133.25,129.62,129.26$, $129.13,119.56,118.98,118.43,105.34,94.65,74.50$, 18.17; MS $m / z$ : $367.1(\mathrm{M}+\mathrm{H})^{+}$. Anal. calcd for $\mathrm{C}_{19} \mathrm{H}_{15^{-}}$ $\mathrm{ClN}_{4} \mathrm{O}_{2}$ : C 62.22, H 4.12, N 15.27; found C 62.15, H 4.11, $\mathrm{N} 15.30$.

2-((1-(4-Chlorophenyl)-1H-pyrazol-3-yl)oxy)- $N$-(4-(trifluoromethyl)phenyl)propanamide (16): $79 \%$ yield. White solid, m.p. $153 \sim 155{ }^{\circ} \mathrm{C}$; ${ }^{1} \mathrm{H}$ NMR $\left(\mathrm{CDCl}_{3}, 300 \mathrm{MHz}\right) \delta$ : $8.44(\mathrm{~s}, 1 \mathrm{H}, \mathrm{NH}), 7.76(\mathrm{~d}, J=2.7 \mathrm{~Hz}, 1 \mathrm{H}$, pyrazol-5-H), $7.71\left(\mathrm{~d}, J=8.4 \mathrm{~Hz}, 2 \mathrm{H}, 4-\mathrm{CF}_{3}-\mathrm{Ph}-3,5-2 \mathrm{H}\right), 7.58$ (d, $J=8.4$ $\left.\mathrm{Hz}, 2 \mathrm{H}, 4-\mathrm{CF}_{3}-\mathrm{Ph}-2,6-2 \mathrm{H}\right), 7.53(\mathrm{~d}, J=8.7 \mathrm{~Hz}, 2 \mathrm{H}$, 4-Cl-Ph-2,6-2H), 7.37 (d, $J=8.7 \mathrm{~Hz}, 2 \mathrm{H}, 4-\mathrm{Cl}-\mathrm{Ph}-3,5-2 \mathrm{H})$, $6.03(\mathrm{~d}, J=2.7 \mathrm{~Hz}, 1 \mathrm{H}$, pyrazol-4-H), 5.27 (q, $J=6.9 \mathrm{~Hz}$, $1 \mathrm{H}, \mathrm{CH}), 1.72\left(\mathrm{~d}, J=6.9 \mathrm{~Hz}, 3 \mathrm{H}, \mathrm{CH}_{3}\right) ;{ }^{13} \mathrm{C} \mathrm{NMR}$ (DMSO- $\left.d_{6}, 150 \mathrm{MHz}\right) \delta: 170.52,162.72,142.27,138.32$, $129.58,129.25,129.13,126.06$ (q, $\left.{ }^{3} J=3.7 \mathrm{~Hz}, \mathrm{~F}-\mathrm{C}\right)$, 123.61 (q, $\left.{ }^{2} J=31.8 \mathrm{~Hz}, \mathrm{~F}-\mathrm{C}\right), 124.33$ (q, ${ }^{1} J=269.70 \mathrm{~Hz}$, F-C), 119.50, 118.44, 94.67, 74.52, 18.22; MS m/z: 410.1 $(\mathrm{M}+\mathrm{H})^{+}$. Anal. calcd for $\mathrm{C}_{19} \mathrm{H}_{15} \mathrm{ClF}_{3} \mathrm{~N}_{3} \mathrm{O}_{2}$ : C 55.69, $\mathrm{H}$ 3.69, N 10.25; found C 55.65, H 3.70, N 10.25.

2-((1-(4-Chlorophenyl)-1H-pyrazol-3-yl)oxy)- $N$-(4-nitrophenyl)propanamide (17): $87 \%$ yield. White solid, m.p. $152 \sim 154{ }^{\circ} \mathrm{C} ;{ }^{1} \mathrm{H}$ NMR $\left(\mathrm{CDCl}_{3}, 300 \mathrm{MHz}\right) \delta: 8.62(\mathrm{~s}, 1 \mathrm{H}$, $\mathrm{NH}), 8.21\left(\mathrm{~d}, J=9.3 \mathrm{~Hz}, 2 \mathrm{H}, 4-\mathrm{NO}_{2}-\mathrm{Ph}-3,5-2 \mathrm{H}\right), 7.76$ (d, $J=3.0 \mathrm{~Hz}, 1 \mathrm{H}$, pyrazol-5-H), 7.77 (d, $J=9.3 \mathrm{~Hz}, 2 \mathrm{H}$, 4-NO $-\mathrm{Ph}-2,6-2 \mathrm{H}$ ), 7.51 (d, $J=8.7 \mathrm{~Hz}, 2 \mathrm{H}, 4-\mathrm{Cl}-\mathrm{Ph}-2,6-$ 2H), 7.40 (d, $J=8.7 \mathrm{~Hz}, 2 \mathrm{H}, 4-\mathrm{Cl}-\mathrm{Ph}-3,5-2 \mathrm{H}), 6.03$ (d, $J=$
$3.0 \mathrm{~Hz}, 1 \mathrm{H}$, pyrazol-4-H), 5.29 (q, $J=6.9 \mathrm{~Hz}, 1 \mathrm{H}, \mathrm{CH})$, $1.72\left(\mathrm{~d}, J=6.9 \mathrm{~Hz}, 3 \mathrm{H}, \mathrm{CH}_{3}\right) ;{ }^{13} \mathrm{C}$ NMR (DMSO- $d_{6}, 150$ MHz) $\delta: 170.86,162.65,144.85,142.45,138.29,129.67$, $129.28,129.12,124.94,119.26,118.42,94.65,74.49$, 18.14; IR v: 3368, 3119, 3050, 2998, 2942, 1696, 1532, 1510, 1335, 1532, 1385, 1092, 824, $743 \mathrm{~cm}^{-1}$; MS m/z: $387.1(\mathrm{M}+\mathrm{H})^{+}$. Anal. calcd for $\mathrm{C}_{18} \mathrm{H}_{15} \mathrm{ClN}_{4} \mathrm{O}_{4}$ : C 55.90, $\mathrm{H}$ 3.91, N 14.49; found C 55.82, H 3.90, N 14.47.

$N$-(4-(tert-Butyl)phenyl)-2-((1-(4-chlorophenyl)-1H-pyrazol-3-yl)oxy)propanamide (18): 78\% yield. White solid, m.p. $154 \sim 156{ }^{\circ} \mathrm{C} ;{ }^{1} \mathrm{H}$ NMR $\left(\mathrm{CDCl}_{3}, 300 \mathrm{MHz}\right) \delta: 8.19$ (s, $1 \mathrm{H}, \mathrm{NH}), 7.75$ (d, $J=2.7 \mathrm{~Hz}, 1 \mathrm{H}$, pyrazol-5-H), 7.54 (d, $J=9.0 \mathrm{~Hz}, 2 \mathrm{H}, 4-\mathrm{Cl}-\mathrm{Ph}-2,6-2 \mathrm{H}), 7.48$ (d, $J=9.0 \mathrm{~Hz}, 2 \mathrm{H}$, 4- $\left.\left(\mathrm{CH}_{3}\right)_{3} \mathrm{CPh}-2,6-2 \mathrm{H}\right), 7.38$ (d, J=9.0 Hz, 2H, 4-Cl-Ph3,5-2H), 7.34 (d, $\left.J=9.0 \mathrm{~Hz}, 2 \mathrm{H}, 4-\left(\mathrm{CH}_{3}\right)_{3} \mathrm{C}-\mathrm{Ph}-3,5-2 \mathrm{H}\right)$, $6.01(\mathrm{~d}, J=2.7 \mathrm{~Hz}, 1 \mathrm{H}$, pyrazol-4-H), $5.22(\mathrm{q}, J=6.6 \mathrm{~Hz}$, $1 \mathrm{H}, \mathrm{CH}), 1.71\left(\mathrm{~d}, J=6.6 \mathrm{~Hz}, 3 \mathrm{H}, \mathrm{CH}_{3}\right), 1.29(\mathrm{~s}, 9 \mathrm{H}$, $\left.3 \mathrm{CH}_{3}\right) ;{ }^{13} \mathrm{C}$ NMR (DMSO- $\left.d_{6}, 150 \mathrm{MHz}\right) \delta: 169.57,162.81$, $145.86,138.36,136.08,129.47,129.26,129.05,125.27$, 119.39, 118.46, 94.70, 74.51, 33.99, 31.16, 18.36; MS m/z: $398.1(\mathrm{M}+\mathrm{H})^{+}$. Anal. calcd for $\mathrm{C}_{22} \mathrm{H}_{24} \mathrm{ClN}_{3} \mathrm{O}_{2}$ : C 66.41, H 6.08, N 10.56; found C 66.35, H 6.08, N 10.57.

2-((1-(4-Chlorophenyl)-1H-pyrazol-3-yl)oxy)- $N$-(2,4-dichlorophenyl)propanamide (19): $82 \%$ yield. White solid, m.p. $141 \sim 143{ }^{\circ} \mathrm{C} ;{ }^{1} \mathrm{H}$ NMR $\left(\mathrm{CDCl}_{3}, 300 \mathrm{MHz}\right) \delta: 8.82$ (s, $1 \mathrm{H}, \mathrm{NH}), 8.41$ (d, $J=8.7 \mathrm{~Hz}, 1 \mathrm{H}, 2,4-2 \mathrm{Cl}-\mathrm{Ph}-6-\mathrm{H}), 7.75$ (d, $J=2.7 \mathrm{~Hz}, 1 \mathrm{H}$, pyrazol-5-H), 7.52 (d, $J=8.7 \mathrm{~Hz}, 2 \mathrm{H}$, 4-Cl-Ph-2,6-2H), 7.37 (d, $J=8.7 \mathrm{~Hz}, 2 \mathrm{H}, 4-\mathrm{Cl}-\mathrm{Ph}-3,5-2 \mathrm{H}$ ), 7.36 (d, $J=2.1 \mathrm{~Hz}, 1 \mathrm{H}, 2,4-2 \mathrm{Cl}-\mathrm{Ph}-3-\mathrm{H}), 7.26$ (dd, ${ }^{3} J=9.0$ $\left.\mathrm{Hz},{ }^{4} J=2.1 \mathrm{~Hz}, 1 \mathrm{H}, 2,4-2 \mathrm{Cl}-\mathrm{Ph}-5-\mathrm{H}\right) ; 6.02(\mathrm{~d}, J=2.7 \mathrm{~Hz}$, 1H, pyrazol-4-H), 5.36 (q, $J=6.9 \mathrm{~Hz}, 1 \mathrm{H}, \mathrm{CH}), 1.73$ (d, $\left.J=6.9 \mathrm{~Hz}, 3 \mathrm{H}, \mathrm{CH}_{3}\right) ;{ }^{13} \mathrm{C} \mathrm{NMR}$ (DMSO-d, $\left.150 \mathrm{MHz}\right) \delta$ : $170.27,163.03,138.72,133.90,130.13,129.98,129.65$, $129.50,129.35,128.17,128.03,127.55,118.88,95.08$, 74.59, 18.71; MS m/z: $410.1(\mathrm{M}+\mathrm{H})^{+}$. Anal. calcd for $\mathrm{C}_{18} \mathrm{H}_{14} \mathrm{Cl}_{3} \mathrm{~N}_{3} \mathrm{O}_{2}$ : C 52.64, H 3.44, N 10.23; found C 52.60, $\mathrm{H} 3.44, \mathrm{~N} 10.24$.

2-((1-(4-Chlorophenyl)-1H-pyrazol-3-yl)oxy)- $N$-(3,4-dichlorophenyl)propanamide (20): 54\% yield. White solid, m.p. $225 \sim 227{ }^{\circ} \mathrm{C} ;{ }^{1} \mathrm{H}$ NMR $\left(\mathrm{CDCl}_{3}, 300 \mathrm{MHz}\right) \delta: 7.73$ $(\mathrm{d}, J=2.7 \mathrm{~Hz}, 1 \mathrm{H}$, pyrazol-5-H), $7.51(\mathrm{~d}, J=8.7 \mathrm{~Hz}, 2 \mathrm{H}$, 4-Cl-Ph-2,6-2H), 7.39 (d, $J=8.7 \mathrm{~Hz}, 1 \mathrm{H}, 3,4-2 \mathrm{Cl}-\mathrm{Ph}-5-\mathrm{H})$, 7.36 (d, $J=8.7 \mathrm{~Hz}, 2 \mathrm{H}, 4-\mathrm{Cl}-\mathrm{Ph}-3,5-2 \mathrm{H}), 7.21$ (d, $J=2.7$ $\mathrm{Hz}, 1 \mathrm{H}, 3,4-2 \mathrm{Cl}-\mathrm{Ph}-2-\mathrm{H}), 6.94$ (dd, ${ }^{3} \mathrm{~J}=8.7 \mathrm{~Hz},{ }^{4} J=2.7$ Hz, 1H, 3,4-2Cl-Ph-6-H), 5.99 (d, $J=2.7$ Hz, 1H, pyrazol4-H), 5.31 (q, $J=6.9 \mathrm{~Hz}, 1 \mathrm{H}, \mathrm{CH}), 1.78$ (d, $J=6.9 \mathrm{~Hz}, 3 \mathrm{H}$, $\left.\mathrm{CH}_{3}\right) ;{ }^{13} \mathrm{C}$ NMR (DMSO- $\left.d_{6}, 150 \mathrm{MHz}\right) \delta: 170.01,162.56$, $149.07,138.25,131.65,131.38,130.02,129.43,129.31$, $128.80,123.90,122.23,118.50,94.49,72.59,17.58$; IR $v$ : 3233, 3103, 2982, 2930, 1677, 1587, 1541, 1495, 1474, 1386, 1093, 829, 815, $713 \mathrm{~cm}^{-1}$; MS m/z: $410.1(\mathrm{M}+\mathrm{H})^{+}$. Anal. calcd for $\mathrm{C}_{18} \mathrm{H}_{14} \mathrm{Cl}_{3} \mathrm{~N}_{3} \mathrm{O}_{2}$ : C 52.64, H 3.44, N 10.23; found $\mathrm{C} 52.57, \mathrm{H} 3.43, \mathrm{~N} 10.22$.

2-((1-(4-Chlorophenyl)-1H-pyrazol-3-yl)oxy)- $N$-(pyridin-3-yl)propanamide (21): $70 \%$ yield. White solid, m.p. 133 $135{ }^{\circ} \mathrm{C} ;{ }^{1} \mathrm{H}$ NMR $\left(\mathrm{CDCl}_{3}, 300 \mathrm{MHz}\right) \delta: 8.61$ (d, $J=$ $2.4 \mathrm{~Hz}, 1 \mathrm{H}, 3$-pyridyl-2-H), 8.42 (s, 1H, NH), 8.37 (dd, 
${ }^{3} J=4.8 \mathrm{~Hz},{ }^{4} J=1.5 \mathrm{~Hz}, 1 \mathrm{H}, 3$-pyridyl-6-H), 8.22 (ddd, ${ }^{3} J=8.4 \mathrm{~Hz},{ }^{4} J=2.4,1.5 \mathrm{~Hz}, 1 \mathrm{H}, 3$-pyridyl-4-H), 7.76 (d, $J=2.7 \mathrm{~Hz}, 1 \mathrm{H}$, pyrazol-5-H), $7.53(\mathrm{~d}, J=9.3 \mathrm{~Hz}, 2 \mathrm{H}$, 4-Cl-Ph-2,6-2H), 7.38 (d, J=9.3 Hz, 2H, 4-Cl-Ph-3,5-2H), $7.28\left(\mathrm{dd},{ }^{3} J=8.4,4.8 \mathrm{~Hz}, 1 \mathrm{H}, 3\right.$-pyridyl-5-H), 6.02 (d, $J=$ $2.7 \mathrm{~Hz}, 1 \mathrm{H}$, pyrazol-4-H), 5.28 (q, $J=6.9 \mathrm{~Hz}, 1 \mathrm{H}, \mathrm{CH})$, $1.72\left(\mathrm{~d}, J=6.9 \mathrm{~Hz}, 3 \mathrm{H}, \mathrm{CH}_{3}\right) ;{ }^{13} \mathrm{C}$ NMR (DMSO- $d_{6}, 150$ MHz) $\delta$ : 170.45, 162.71, 144.53, 141.24, 138.33, 135.31, $129.60,129.29,129.11,126.60,123.61,118.45,94.70$, 74.47, 18.24; IR v: 3247, 3185, 3125, 3072, 2984, 2936, 1672, 1599, 1539, 1499, 1475, 1375, 1269, 1092, 832, 749, $706 \mathrm{~cm}^{-1}$; MS m/z: $343.1(\mathrm{M}+\mathrm{H})^{+}$. Anal. calcd for $\mathrm{C}_{17} \mathrm{H}_{15^{-}} \mathrm{ClN}_{4} \mathrm{O}_{2}$ : C 59.57, H 4.41, N 16.35; found C 59.49, $\mathrm{H} 4.40, \mathrm{~N} 16.34$.

2-((1-(4-Chlorophenyl)-1H-pyrazol-3-yl)oxy)- $N$-(pyridin-2-yl)propanamide (22): $69 \%$ yield. Pale yellow oil. ${ }^{1} \mathrm{H}$ $\mathrm{NMR}\left(\mathrm{CDCl}_{3}, 300 \mathrm{MHz}\right) \delta: 8.88(\mathrm{~s}, 1 \mathrm{H}, \mathrm{NH}), 8.29 \sim 8.26$ (m, 2H, 2-pyridyl-3,6-2H), 7.75 7.69 (m, 2H, 2-pyridyl4-H; pyrazol-5-H), 7.52 (d, $J=9.0 \mathrm{~Hz}, 2 \mathrm{H}, 4-\mathrm{Cl}-\mathrm{Ph}-2,6-$ 2H), 7.36 (d, $J=9.0 \mathrm{~Hz}, 2 \mathrm{H}, 4-\mathrm{Cl}-\mathrm{Ph}-3,5-2 \mathrm{H}), 7.08 \sim 7.03$ (m, 1H, 2-pyridyl-5-H), 6.00 (d, $J=2.7 \mathrm{~Hz}, 1 \mathrm{H}$, pyrazol4-H), 5.33 (q, $J=6.6 \mathrm{~Hz}, 1 \mathrm{H}, \mathrm{CH}), 1.72$ (d, $J=6.6 \mathrm{~Hz}, 3 \mathrm{H}$, $\left.\mathrm{CH}_{3}\right) ;{ }^{13} \mathrm{C}$ NMR $\left(150 \mathrm{MHz}, \mathrm{DMSO}-d_{6}\right) \delta: 170.62,162.77$, $151.62,148.07,138.34,138.25,129.56,129.22,129.05$, 119.70, 118.44, 113.64, 94.57, 73.86, 18.34; IR v: 3399, 3123, 3076, 2984, 2935, 1692, 1595, 1577, 1541, 1516, $1500,1470,1387,1295,1091,825,776,739 \mathrm{~cm}^{-1}$; MS $m / z: 343.2(\mathrm{M}+\mathrm{H})^{+}$. Anal. calcd for $\mathrm{C}_{17} \mathrm{H}_{15} \mathrm{ClN}_{4} \mathrm{O}_{2}: \mathrm{C}$ 59.57, H 4.41, N 16.35; found C 59.50, H 4.40, N 16.39.

2-((1-(4-Chlorophenyl)-1H-pyrazol-3-yl)oxy)- $N$-(3-methylpyridin-2-yl)propanamide (23): 74\% yield. White solid, m.p. $136 \sim 138{ }^{\circ} \mathrm{C} ;{ }^{1} \mathrm{H}$ NMR $\left(\mathrm{CDCl}_{3}, 300 \mathrm{MHz}\right) \delta$ : 8.51 (s, $1 \mathrm{H}, \mathrm{NH}), 8.29$ (dd, ${ }^{3} J=4.8 \mathrm{~Hz},{ }^{4} J=1.2 \mathrm{~Hz}, 1 \mathrm{H}, 3$-methylpyridin-2-yl-6-H), 7.75 (d, $J=2.7 \mathrm{~Hz}, 1 \mathrm{H}$, pyrazol-5-H), $7.56 \sim 7.53(\mathrm{~m}, 3 \mathrm{H}, 4-\mathrm{Cl}-\mathrm{Ph}-2,6-2 \mathrm{H}, 3-$ methylpyridin2-yl-4-H), 7.37 (d, $J=9.0 \mathrm{~Hz}, 2 \mathrm{H}, 4-\mathrm{Cl}-\mathrm{Ph}-3,5-2 \mathrm{H}$ ), 7.11 (dd, ${ }^{3} J=7.8,4.8 \mathrm{~Hz}, 1 \mathrm{H}, 3-$ methylpyridin-2-yl-5-H), 6.00 (d, $J=2.7 \mathrm{~Hz}, 1 \mathrm{H}$, pyrazol-4-H), 5.32 (q, 6.9 Hz, 1H, CH), $2.21\left(\mathrm{~s}, 3 \mathrm{H}, \mathrm{CH}_{3}\right), 1.75\left(\mathrm{~d}, J=6.9 \mathrm{~Hz}, 3 \mathrm{H}, \mathrm{CH}_{3}\right)$; $\mathrm{MS} m / z$ : $357.2(\mathrm{M}+\mathrm{H}){ }^{+} ;{ }^{13} \mathrm{C}$ NMR (150 MHz, DMSO-d $) \delta$ : $170.02,162.81,162.81,149.53,145.86,139.26,138.37$, $129.42,129.21,129.03,121.95,118.58,94.73,73.95$, 18.47, 17.35; MS m/z: $357.2(\mathrm{M}+\mathrm{H})^{+}$. Anal. calcd for $\mathrm{C}_{18} \mathrm{H}_{17} \mathrm{ClN}_{4} \mathrm{O}_{2}$ : C 60.59, $\mathrm{H} 4.80, \mathrm{~N} 15.70$; found $\mathrm{C} 60.50$, H 4.79, N 15.69.

2-((1-(4-Chlorophenyl)-1H-pyrazol-3-yl)oxy)- $N$-(6-methylpyridin-2-yl)propanamide (24): 77\% yield. Pale yellow oil. ${ }^{1} \mathrm{H}$ NMR $\left(\mathrm{CDCl}_{3}, 300 \mathrm{MHz}\right) \delta: 8.83$ (s, 1H, NH), 8.08 (d, $J=7.5 \mathrm{~Hz}, 1 \mathrm{H}, 6$-methylpyridin-2-yl-3-H), 7.72 (d, $J=$ $2.4 \mathrm{~Hz}, 1 \mathrm{H}$, pyrazol-5-H), 7.60 (dd, ${ }^{3} J=7.5,7.5 \mathrm{~Hz}, 1 \mathrm{H}, 6-$ methylpyridin-2-yl-4-H), 7.53 (d, $J=8.7 \mathrm{~Hz}, 2 \mathrm{H}, 4-\mathrm{Cl}-\mathrm{Ph}-$ 2,6-2H), 7.35 (d, $J=8.7 \mathrm{~Hz}, 2 \mathrm{H}, 4-\mathrm{Cl}-\mathrm{Ph}-3,5-2 \mathrm{H}), 6.91$ (d, $J=7.5 \mathrm{~Hz}, 1 \mathrm{H}, 6-$ methylpyridin-2-yl-5-H), 6.01 (d, $J=2.4$ $\mathrm{Hz}, 1 \mathrm{H}$, pyrazol-4-H), 5.30 (q, $J=6.9 \mathrm{~Hz}, 1 \mathrm{H}, \mathrm{CH}$ ), 2.46 $\left(\mathrm{s}, 3 \mathrm{H}, \mathrm{CH}_{3}\right), 1.71\left(\mathrm{~d}, J=6.9 \mathrm{~Hz}, 3 \mathrm{H}, \mathrm{CH}_{3}\right) ;{ }^{13} \mathrm{C} \mathrm{NMR}$ (DMSO- $\left.d_{6}, 150 \mathrm{MHz}\right) \delta: 170.50,162.79,156.59,150.95$, 138.52 , 138.34, 129.50, 129.19, 129.04, 118.87, 118.46,
110.50, 94.54, 73.85, 23.55, 18.37; MS m/z: $357.2(\mathrm{M}+$ $\mathrm{H})^{+}$. Anal. calcd for $\mathrm{C}_{18} \mathrm{H}_{17} \mathrm{ClN}_{4} \mathrm{O}_{2}: \mathrm{C} 60.59, \mathrm{H} 4.80, \mathrm{~N}$ 15.70; found $\mathrm{C} 60.52, \mathrm{H} 4.78 \mathrm{~N} 15.72$.

2-((1-(4-Chlorophenyl)-1H-pyrazol-3-yl)oxy)- $N$-(6-chloropyridazin-3-yl)propanamide (25): $76 \%$ yield. White solid, m.p. $149 \sim 151{ }^{\circ} \mathrm{C} ;{ }^{1} \mathrm{H}$ NMR $\left(\mathrm{CDCl}_{3}, 300 \mathrm{MHz}\right) \delta$ : $9.38(\mathrm{~s}, 1 \mathrm{H}, \mathrm{NH}), 8.56(\mathrm{~d}, J=9.3 \mathrm{~Hz}, 1 \mathrm{H}, 6$-chloropyridazin-3-yl-4-H), 7.74 (d, $J=2.7 \mathrm{~Hz}, 1 \mathrm{H}$, pyrazol-5-H), $7.52 \sim 7.50$ (m, 3H, 4-Cl-Ph-2,6-2H; 6-chloropyridazin3-yl-5-H), 7.37 (d, $J=8.7$ Hz, 2H, 4-Cl-Ph-3,5-2H), 6.03 (d, $J=2.7 \mathrm{~Hz}, 1 \mathrm{H}$, pyrazol-4-H), 5.38 (q, $J=6.9 \mathrm{~Hz}, 1 \mathrm{H}$, $\mathrm{CH}), 1.73\left(\mathrm{~d}, J=6.9 \mathrm{~Hz}, 3 \mathrm{H}, \mathrm{CH}_{3}\right) ;{ }^{13} \mathrm{C}$ NMR (DMSO- $d_{6}$, $150 \mathrm{MHz}) \delta: 171.70,16.66,154.97,151.35,138.29$, $130.31,129.67,129.25,129.07,121.59,118.40$, 94.54, 73.69, 18.19; MS m/z: $378.1(\mathrm{M}+\mathrm{H})^{+}$. Anal. calcd for $\mathrm{C}_{16} \mathrm{H}_{13} \mathrm{Cl}_{2} \mathrm{~N}_{5} \mathrm{O}_{2}$ : C 50.81, H 3.46, $\mathrm{N} \mathrm{18.52;} \mathrm{found,} \mathrm{C} \mathrm{50.75,}$ $\mathrm{H} 3.45, \mathrm{~N} 18.54$.

\subsection{Fungicidal assay}

The antifungal activities in vitro of all the synthesized compounds against Rhizoctonia solani Kühn were conducted by the mycelium linear growth rate method as previously reported. ${ }^{[27 \sim 29]}$ The details are as follows: each of the tested compounds $(30 \mathrm{mg})$ was completely dissolved in $30 \mathrm{~mL}$ of acetone/methanol $(V: V=1: 1)$, appropriate water containing $0.1 \%$ Tween 80 was then added to generate the stock solution. At the same time, the same volume of acetone/methanol $(V: V=1: 1)$ was diluted by water containing $0.1 \%$ Tween 80 as blank control. $1 \mathrm{~mL}$ of the resulting solution was added to $14 \mathrm{~mL}$ of melted PDA agar at $45 \sim 50{ }^{\circ} \mathrm{C}$. After quickly and completely mixing, the above mixture was poured into a Petri dish in a laminar flow chamber. The final concentration of the test compound in the culture medium was $100,50,25,12.5,6.25$ and $3.125 \mathrm{mg} / \mathrm{L}$. When the medium in the plate was partially solidified, a $5 \mathrm{~mm}$ thick and $5 \mathrm{~mm}$ diameter disc of fungus (Rhizoctonia solani Kühn) cut from sub-cultured Petri dishes ahead of time was placed at the center of the semisolid medium. The dishes were kept in an incubator at $(25 \pm 1){ }^{\circ} \mathrm{C}$ for $5 \sim 7 \mathrm{~d}$ (taking full growth of bacteria as standard). Each experiment was carried out in triplicate. The diameters (in $\mathrm{mm}$ ) of inhibition zones were measured with cross-bonded methods, and the growth inhibition rates were calculated according to the following formula:

Inhibition rate $(\%)=\left[\left(d_{\mathrm{c}}-d_{0}\right)-\left(d_{\mathrm{s}}-d_{0}\right)\right] /\left(d_{\mathrm{c}}-d_{0}\right) \times 100 \%$

Wherein: $d_{0}$ is the diameter of the fungus cut, $d_{\mathrm{c}}$ is the diameter of a fungal colony in the blank test, and $d_{\mathrm{s}}$ is the diameter of a fungal colony in the compound-treated test.

The $\mathrm{EC}_{50}$ values were calculated by Duncan's new multiple range method using DPS version 14.5. The test results of the fungicidal activities of compounds $\mathbf{1} \sim \mathbf{2 5}$ are listed in Tables $1 \sim 3$.

Supporting information ${ }^{1} \mathrm{H}$ NMR, ${ }^{13} \mathrm{C}$ NMR, IR and MS spectra for the synthesized compounds. The Supporting Information is available free of charge via the Internet 
at http://sioc-journal.cn.

\section{References and note}

[1] Gu, A. L.; Bai, Y. L. Mod. Agrochem. 2018, 17, 1 (in Chinese). (顾安乐, 柏亚罗, 现代农药, 2018, 17, 1.)

[2] Yu, G. P. M.S. Thesis, Chinese Academy of Agricultural Sciences, Beijing, 2009 (in Chinese). (虞国平，硕士论文，中国农业科学院，北京, 2009.)

[3] Gong, T. T. Ph.D. Dissertation, Minzu University of China, Beijing, 2017 (in Chinese). (公婷婷, 博士论文, 中央民族大学, 北京, 2017.)

[4] Wang, J. G.; Chen, H. S.; Zhao, W. G.; Ma, Y.; Li, Z. M.; Han, Y. F. Chin. J. Org. Chem. 2002, 22, 2043 (in Chinese). (王建国, 陈寒松, 赵卫光, 马翼, 李正名, 韩玉芬, 有机化学, 2002, 22, 2043.)

[5] Meng, L. M. S. Thesis, Nanjing Agricultural University, Nanjing, 2013 (in Chinese). (孟琳，硕士论文，南京农业大学，南京, 2013.)

[6] Gui, W. X. Anhui Agric. Sci. Bull. 2012, 18, 109 (in Chinese). (桂文信, 安徽农学通报, 2012, 18, 109.)

[7] Leng, P.; Yuan, L.; Wei, P.; Lu. S. Q.; Zhang, X. F. Anhui Agric. Sci. Bull. 2012, 18, 92 (in Chinese).

(冷鹏, 袁蕾, 魏萍, 陆书桥, 张绪芬, 安徽农学通报, 2012, 18, 92.)

[8] Ma, H. J.; Cao, Q. L.; Ma, Y. F.; Ni, J. P. Chin. J. Pestic. 2013, 52, 408 (in Chinese). (马海军, 曹庆亮, 马亚芳, 倪玨萍, 农药, 2013, 52, 408.)

[9] Sun, X. M.S. Thesis, Jilin Agricultural University, Changchun, 2015 (in Chinese). (孙雪, 硕士论文, 吉林农业大学, 长春, 2015.)

[10] Wang, Q. S.; Li, L. J.; Cai, Z. Y. Ye, G. W. World Pestic. 2017, 39, 55 (in Chinese). (汪青松，李俐杰，蔡真瑜，叶光武，世界农药, 2017, 39, 55.)

[11] Meng, Y. T. Anhui Agri. Sci. Bull. 2015, 21(16), 74 (in Chinese). (孟叶挺, 安徽农学通报, 2015, 21(16), 74.)

[12] Guan, A. Y.; Liu, C. L.; Chen, W.; Yang, F.; Xie, Y.; Zhang, J. B.; Li, Z. N.; Wang, M. A. J. Agric. Food Chem. 2017, 65, 1272.

[13] Moon, J. K.; Keum, Y. S.; Hwang, E. C.; Park, B. S.; Chang, H. R.; Li, Q. X.; Kim, J. H. J. Agric. Food Chem. 2007, 55, 5416.

[14] Zeng, Z. W.; Jiang, Y. J. Chin. J. Pestic. 2004, 43, 327 (in Chi- nese).

(曾仲武，姜雅君，农药, 2004, 43, 327.)

[15] MacBean, C. The Pesticide Manual, 16th ed., BCPC, Alton, UK, 2012, p. 264.

[16] Zhu, G. L.; Yao, R. S.; Zhu, H. X.; Wang, H. Biotechnol. Lett. 2012, 34, 709 .

[17] Yang, Z. H.; Chen, A. Y.; Hu, A. X.; Ye, J. Chin. J. Org. Chem. 2017, 37, 149 (in Chinese).

(杨子辉，陈爱羽，胡艾希，叶姣，有机化学， 2017, 37, 149.)

[18] Hemmi, S.; Usui, S.; Hatamoto, M.; Fukuchi, T. WO 2017213137, 2017 [Chem. Abstr. 2017, 168, 76494].

[19] Liu, C. L.; Zhou, Y. P.; Li, Z. N.; Liu, Y. X.; Zhang, H. CN 101747263, 2010 [Chem. Abstr. 2010, 153, 145329].

[20] Huang, Z. Q.; Feng, C. P.; Shi, J.; Lv, L. J.; Bu, L. X.; Yang, R. J.; Wang, Y. H. CN 106632083, 2017 [Chem. Abstr. 2017, 167, 31057].

[21] MacBean, C. The Pesticide Manual, 16th ed., BCPC, Alton, UK, 2012, p. 963.

[22] Liu, C. L. Chin. J. Pestic. 2011, 50, 20 (in Chinese). (刘长令, 农药, 2011, 50, 20.)

[23] Guan, A. Y.; Liu, C. L.; Yang, X. P.; Dekeyser, M. Chem. Rev. 2014, 114, 7079.

[24] Liu, C. L.; Guan, A. Y.; Yang, J. D.; Chai, B. S.; Li, M.; Li, H. C.; Yang, J. C.; Xie, Y. J. Agric. Food Chem. 2016, 64, 45.

[25] The atomic coordinates for compound $\mathbf{8}$ have been deposited at the Cambridge Crystallographic Data Centre. CCDC ID: 1537182 contain the supplementary crystallographic data for this Article. These data can be obtained free of charge from The Cambridge Crystallographic Data Centre via www.ccdc.cam.ac.uk/data_re- quest/cif).

[26] Yu, F. Q.; Guan, A. Y.; Li, M. R.; Hu, L.; Li, X. W. Chin. Chem. Lett. 2018, 29, 915.

[27] Yang, R.; Gao, Z. F.; Zhao, J. Y.; Li, W. B.; Zhou, L.; Miao, F. J. Agric. Food Chem. 2015, 63, 1906.

[28] Liu, Y.; Liu, M.; Chen, M. G.; Wu, C. C.; Hua, X. W.; Zhou, S.; Wang, B. L.; Li, Z. M. Chin. J. Org. Chem. 2017, 37, 403 (in Chinese). (刘阳，刘明，陈明桂，吴长春，华学文，周莎，王宝雷，李正名， 有机化学, 2017, 37, 403.)

[29] Qiao, L. L.; Wei, Y.; Hao, S. H. Chin. J. Org. Chem. 2018, 38, 509 (in Chinese)

(乔丽丽, 魏艳, 郝双红, 有机化学, 2018, 38, 509.)

(Li, L.; Fan, Y.) 